\title{
Off-shell vs on-shell modelling of top quarks in photon associated production
}

\author{
G. Bevilacqua, ${ }^{a}$ H.B. Hartanto, ${ }^{b}$ M. Kraus, ${ }^{c}$ T. Weber ${ }^{d}$ and M. Worek ${ }^{d}$ \\ ${ }^{a}$ MTA-DE Particle Physics Research Group, University of Debrecen, \\ H-4010 Debrecen, PBox 105, Hungary \\ ${ }^{b}$ Institute for Particle Physics Phenomenology, Department of Physics, \\ Durham University, Durham, DH1 3LE, U.K. \\ ${ }^{c}$ Physics Department, Florida State University, \\ Tallahassee, FL 32306-4350, U.S.A. \\ ${ }^{d}$ Institute for Theoretical Particle Physics and Cosmology, \\ RWTH Aachen University, D-52056 Aachen, Germany \\ E-mail: giuseppe.bevilacqua@science.unideb.hu, \\ heribertus.b.hartanto@durham.ac.uk, mkraus@hep.fsu.edu, \\ tweber@physik.rwth-aachen.de, worek@physik.rwth-aachen.de
}

ABSTRACT: We present a comparative study of various approaches for modelling of the $e^{+} \nu_{e} \mu^{-} \bar{\nu}_{\mu} b \bar{b} \gamma$ final state in $t \bar{t} \gamma$ production at the LHC. Working at the NLO in QCD we compare the fully realistic description of the top quark decay chain with the one provided by the narrow-width-approximation. The former approach comprises all double, single and non-resonant diagrams, interferences, and off-shell effects of the top quarks. The latter incorporates only double resonant contributions and restricts the unstable top quarks to on-shell states. We confirm that for the integrated cross sections the finite top quark width effects are small and of the order of $\mathcal{O}\left(\Gamma_{t} / m_{t}\right)$. We show, however, that they are strongly enhanced for more exclusive observables. In addition, we investigate fractions of events where the photon is radiated either in the production or in the decay stage. We find that large fraction of isolated photons comes from radiative decays of top quarks. Based on our findings, selection criteria might be developed to reduce such contributions, that constitute a background for the measurement of the anomalous couplings in the $t \bar{t} \gamma$ vertex.

KeYwords: NLO Computations, QCD Phenomenology

ARXiv EPrint: 1912.09999 


\section{Contents}

1 Introduction 1

2 The narrow width approximation $\quad 4$

2.1 Implementing the NWA in HELAC-NLO 6

$\begin{array}{lll}2.2 & \text { Virtual corrections } & 7\end{array}$

$\begin{array}{lll}2.3 & \text { Real corrections } & 7\end{array}$

$\begin{array}{lll}2.4 & \text { Numerical checks } & 10\end{array}$

$\begin{array}{lll}3 & \text { LHC setup } & 11\end{array}$

4 Stability of the full off-shell result $\quad 13$

5 Phenomenological results for integrated cross sections 14

6 Phenomenological results for differential cross sections $\quad 17$

$\begin{array}{lll}6.1 & \text { Off-shell vs. on-shell top quark modelling } & 17\end{array}$

6.2 Double-, single- and non-resonant phase-space regions 21

6.3 Photon radiation in the production and decays 24

$\begin{array}{llr}7 & \text { Conclusions } & 26\end{array}$

\section{Introduction}

Higher order predictions for top quark pair production allow us to deepen our understanding of the Standard Model (SM). By carefully studying with very high accuracy the properties of the heaviest particle discovered so far, physicists might shed some light on physics beyond the SM, so sought-after at the LHC. Besides $t \bar{t}(j)$ production, however, more exclusive $t \bar{t} V, V=\gamma, Z, H, W^{ \pm}$final states are produced and thoroughly analysed at the LHC. Even though $t \bar{t} V$ cross sections are orders of magnitude smaller than those of $\sigma_{p p \rightarrow t \bar{t}}$ and $\sigma_{p p \rightarrow t \bar{t} j}$, they add greatly to the already rich top quark research plans, which are carried out by the ATLAS and CMS collaborations. In particular, among all associated processes the associated production of top quark pairs with a photon has the highest production rate at the LHC. First evidence for $t \bar{t} \gamma$ was reported by the CDF collaboration [1], whereas the observation of the process with a significance of $5.3 \sigma$ was established at $\sqrt{s}=7 \mathrm{TeV}$ by the ATLAS collaboration [2]. Both ATLAS and CMS measured $t \bar{t} \gamma$ cross section at $\sqrt{s}=8 \mathrm{TeV}[3,4]$. But only recently first measurements of the differential cross sections at $13 \mathrm{TeV}$ have been performed by the ATLAS collaboration [5, 6].

The $t \bar{t} \gamma$ process probes the $t \gamma$ electroweak coupling, thus, provides a direct way to measure the top quark electric charge [7]. The latter is known to be $Q_{t}=+2 / 3$, i.e. 
consistent with the SM, albeit has only been measured indirectly in $t \bar{t}$ production $[8,9]$. More exotic physics scenarios, that propose a heavy quark of electric charge $Q_{t}=-4 / 3$, instead of the SM top quark, have been excluded with a significance of more than $8 \sigma$. Not only the strength but also the structure of $t \bar{t} \gamma$ vertex can be examined with the help of the $p p \rightarrow t \bar{t} \gamma$ production process. Based on the fundamental principle of gauge symmetry, the $t \bar{t} \gamma$ vertex, which includes the SM coupling given by the top-quark electric charge $Q_{t}$, and contributions from dimension-six effective operators, can be parametrised using only $\gamma^{\mu}$ and $\sigma^{\mu \nu} q_{\nu}$, where $q=\left(p_{\bar{t}}-p_{t}\right)$ is the outgoing photon momentum [10]. Because the $\gamma^{\mu}$ term does not receive corrections from dimension-six gauge invariant operators the electroweak top anomalous interactions can be described in terms of only two anomalous couplings (two Wilson coefficients) that are the coefficients of the effective $\sigma^{\mu \nu} q_{\nu}$ interactions. The latter should be constrained at the LHC already at $13 \mathrm{TeV}$ with an integrated luminosity of about $300 \mathrm{fb}^{-1}[11-14]$.

Furthermore, production of top quark pairs in association with a photon can be employed to obtain predictions for integrated and differential cross section ratios [15], that are defined according to

$$
\mathcal{R}=\frac{\sigma_{t \bar{t} \gamma}}{\sigma_{t \bar{t}}}, \quad \text { and } \quad \mathcal{R}_{X}=\left(\frac{d \sigma_{t \bar{t} \gamma}}{d X}\right)\left(\frac{d \sigma_{t \bar{t}}}{d X}\right)^{-1},
$$

where $X$ stands for the kinematic observable under consideration (e.g. $M_{b \bar{b}}, M_{\ell \ell}, \Delta \phi_{\ell \ell}$ ). The cross section ratios have many advantages compared to absolute cross sections. They are, for example, more stable against radiative corrections and have reduced scale dependence. Considering that $t \bar{t} \gamma$ and $t \bar{t}$ are very similar processes from the QCD point of view many theoretical and experimental uncertainties cancel in ratio. Consequently, $\mathcal{R}$ and $\mathcal{R}_{X}$ have enhanced predictive power and are interesting not only to study the $t \bar{t} \gamma$ process with the highest precision that until now has only been reserved for the top quark pair production at NNLO in QCD, but also to probe new physics. The latter might reveal itself once sufficiently precise theoretical predictions are compared with appropriately precise experimental data.

Finally, the $t \bar{t}$ charge asymmetry, $A_{C}$, the di-leptonic charge asymmetry, $A_{C}^{\ell \ell}$, as well as, the laboratory frame single asymmetry, $A_{C}^{t \ell}$, and the single lepton asymmetry defined in the $t \bar{t}$ rest frame, $A_{C}^{\ell}$, can be investigated in $t \bar{t} \gamma$ production at the LHC and the high luminosity LHC. They provide complementary information to the measured asymmetries in $t \bar{t}$ production [16-18].

To be able to provide reliable and very precise theoretical predictions for the $p p \rightarrow t \bar{t} \gamma$ production process higher order effects in $\alpha_{s}$ must be incorporated. NLO QCD corrections to the inclusive $t \bar{t} \gamma$ production with on-shell top quarks have been calculated for the first time in ref. [19] and afterwards recomputed in refs. [20, 21]. In ref. [22] results with NLO electroweak corrections have been provided. In all these cases, however, top quarks were treated as stable particles. Such predictions may give us a general idea of the size of the NLO corrections. Because they lack top quark decays, however, they are neither capable to ensure a reliable description of the fiducial phase space regions nor to give us a glimpse into the top quark radiation pattern. For more realistic studies top quark decays 
are needed. First attempt in this direction has been carried out in ref. [23] where NLO QCD theoretical predictions for stable top quarks and a hard photon have been matched with parton shower (PS) Monte Carlo (MC) programs using the PowHel framework. The PowHel approach relies on the Powheg-Box system $[24,25]$ and allows for the matching between fixed order computation at NLO in QCD and parton shower simulation, followed by hadronisation and hadronic decays. The former is provided by the HELAC-NLO MC program [26] and the latter by the general purpose MC program like PYTHIA [27] or HeRwig [28]. In ref. [23] top quark decays have been treated in the PS approximation omitting $t \bar{t}$ spin correlations and photon emission in parton shower evolution. First fully realistic theoretical predictions for $t \bar{t} \gamma$ have been presented in ref. [29] where top quark decays in the narrow width approximation (NWA) have been included, maintaining spin correlations of final state particles. In addition, photon radiation off top quark decay products has been incorporated. They brought along a significant contribution to the cross section. Finally, in ref. [30] a complete description of top quark pair production in association with a hard photon in the di-lepton top quark decay channel has been presented. It is based on matrix elements for $e^{+} \nu_{e} \mu^{-} \nu_{\mu} b \bar{b} \gamma$ production and included all resonant and non-resonant diagrams, interferences, and off-shell effects of the top quarks and the $W$ gauge bosons. This calculation constituted the first full computation for the $t \bar{t} \gamma$ production process at NLO in QCD.

Having different theoretical approaches available for the modelling of top quark decays, it is only natural to investigate whether the full result is always mandatory for the description of various observables. In other words when it might be safe to replace the full result by the one from the NWA. The goal of this paper is, therefore, to compare these two approaches that we shall refer to as $N W A$ and full off-shell. Furthermore, we shall approximate the NLO+PS results featured in ref. [23] by the ones in NWA that keeps the isolated photon emission only in the production stage and allows only LO top quark decays. For brevity, we will refer to this last approach as $\mathrm{NWA}_{\text {LOdecay }}$. Another motivation for the paper is more theoretical. We shall carry out the systematic comparison within one framework. To this end we have implemented the full NWA for top quark related processes into Helac-Nlo. This required substantial modifications of both parts of the program: Helac-1Loop [31] and Helac-Dipoles [32] and an inclusion of an additional polarised dipole into our implementation of the Catani-Seymour subtraction scheme. Having a fully flexible MC program with both options allows us to investigate the fraction of events with photon radiation in the production and in the decays and compare double-, single- and non-resonant contributions of the full off-shell result to the NWA.

The rest of the paper is organised as follows. In section 2 details of the NWA implementation into the HELAC-NLO system are shortly outlined. In section 3 the SM input parameters and the cuts on final states are listed. Stability of the full off-shell result with respect to the transverse momentum cut on the bottom jet is examined in section 4 . Numerical results for the integrated cross sections are presented in section 5, while various differential cross sections are studied in section 6 . Finally, in section 7 we give our conclusions. 


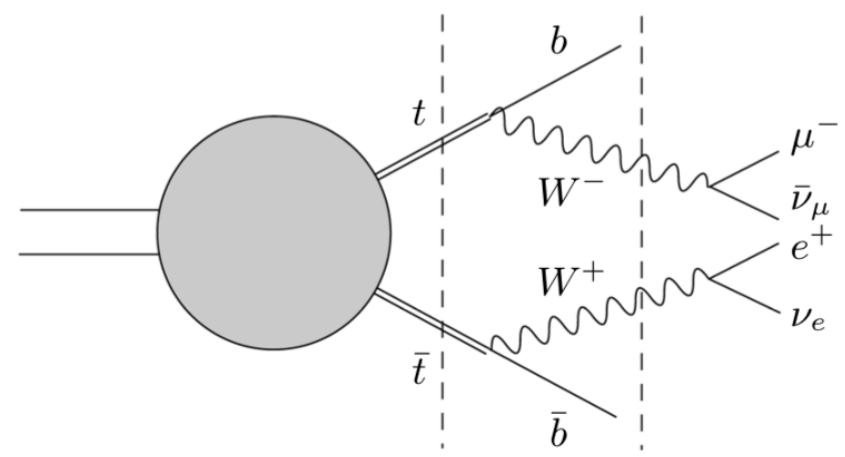

level $0 \quad$ level $1 \quad$ level 2

Figure 1. Schematic illustration of the decay chain for the $p p \rightarrow t \bar{t} \rightarrow b \bar{b} W^{+} W^{-} \rightarrow b \bar{b} e^{+} \nu_{e} \mu^{-} \bar{\nu}_{\mu}$ process. The dashed lines indicate top quark and $W$ gauge boson propagators which are treated as on-shell particles in the NWA.

\section{The narrow width approximation}

The NWA offers a conceptually easy and powerful method for computing cross sections for processes comprising the unstable resonances when the width $(\Gamma)$ of the unstable particle is much smaller than its mass $(M)$, see e.g. [33, 34]. Therefore, for the top quark the NWA allows to make predictions for realistic final states described in terms of light and $b$-jets, charged leptons as well as missing transverse momentum and gives the opportunity for direct comparisons with cross section measurements in the fiducial phase space regions. Prominent examples include the calculations for the $p p \rightarrow t \bar{t}(j)$ process at NLO QCD and theoretical predictions for $t \bar{t}$ at NNLO in QCD [35-39]. The NWA is well established and allows factorisation of the cross section into production times decays due to the following relation

$$
\frac{1}{\left(p_{t}^{2}-m_{t}^{2}\right)^{2}+m_{t}^{2} \Gamma_{t}^{2}} \stackrel{\Gamma_{t} / m_{t} \rightarrow 0}{\longrightarrow} \frac{\pi}{m_{t} \Gamma_{t}} \delta\left(p_{t}^{2}-m_{t}^{2}\right)+\mathcal{O}\left(\frac{\Gamma_{t}}{m_{t}}\right)
$$

where $\Gamma_{t}$ and $m_{t}$ are the width and mass of the top quark. All effects related to the off-shellness of the top quarks as well as non-resonant contributions are systematically neglected in the computation of scattering amplitudes. The neglected contributions are suppressed by the $\Gamma_{t} / m_{t}$ ratio for sufficiently inclusive observables [40], although they can be enhanced for various differential cross sections. In specific phase space regions, like in the high $p_{T}$ region of various dimensionful observables or in the vicinity of kinematical thresholds and edges, they contribute up to $20 \%-50 \%$ [41, 42]. Although there is no doubt that the full off-shell calculations should be used if available, as they provide the most realistic description of the processes under consideration, they are computationally demanding and have practical limitations already at the NLO in perturbation theory. For example, obtaining NLO QCD predictions with full off-shell effects for the $t \bar{t} t \bar{t}$ production process is currently very difficult to imagine. 
In the $\Gamma_{t} / m_{t} \rightarrow 0$ limit, the differential cross-section for the top quark pair production in the di-lepton decay channel is given by

$$
d \sigma_{t \bar{t}}^{\mathrm{NWA}}=d \sigma_{t \bar{t}} d \mathcal{B}_{t \rightarrow b e^{+} \nu_{e}} d \mathcal{B}_{\bar{t} \rightarrow \bar{b} \mu^{-} \bar{\nu}_{\mu}}
$$

where $\mathcal{B}$ stands for the respective branching fraction. The above equation is valid to all orders in the strong coupling constant. A graphical representation is given in figure 1 where both top quarks and $W$ bosons are treated in the NWA and the decay chain is structured into three levels; level 0 - the production process of $t \bar{t}$, level 1 and finally 2 - the fully decayed di-lepton final state. The factorisation for $t \bar{t} \gamma$ can be written in the similar manner by inserting a photon in either of the three terms, giving rise to the following non-overlapping resonant structures, see e.g. ref. [29]

$$
\begin{aligned}
d \sigma_{t \bar{t} \gamma}^{\mathrm{NWA}}= & d \sigma_{t \bar{t} \gamma} d \mathcal{B}_{t \rightarrow b e^{+} \nu_{e}} d \mathcal{B}_{\bar{t} \rightarrow \bar{b} \mu^{-} \bar{\nu}_{\mu}} \\
& +d \sigma_{t \bar{t}}\left(d \mathcal{B}_{t \rightarrow b e^{+} \nu_{e} \gamma} d \mathcal{B}_{\bar{t} \rightarrow \bar{b} \mu^{-} \bar{\nu}_{\mu}}+d \mathcal{B}_{t \rightarrow b e^{+} \nu_{e}} d \mathcal{B}_{\bar{t} \rightarrow \bar{b} \mu^{-} \bar{\nu}_{\mu} \gamma}\right)
\end{aligned}
$$

Accordingly, $t \bar{t} \gamma$ production can be seen as described by two distinct kinematics. On one hand we have photon emission in the production part of the process and on the other hand off the top quark decay products. Although computational complexity is much lower than for the full off-shell calculation, the number of contributions that need to be calculated for $t \bar{t} \gamma$ production in NWA increases rapidly. The same applies to gluon radiation, which must naturally be taken into account when NLO QCD corrections are calculated. Thus, generally speaking the NWA approach is characterised by a proliferation of the contributions that need to be put together to account for all possible resonant structures of $d \sigma_{t \bar{t} \gamma}^{\mathrm{NWA}}$. Several key processes, that are relevant for top quark physics at the LHC, have already been computed in the NWA approach. Nevertheless a fully flexible MC generator capable of performing automated predictions in the NWA at NLO in QCD for all $t \bar{t}$ plus additional object $\left(X=\gamma, Z, W^{ \pm}, H, \ldots\right)$ processes is still missing, even-though results for a variety of $t \bar{t} X$ processes exist in the literature. ${ }^{1}$ Such a MC program should include NLO QCD corrections to $t \bar{t} X$ production and top quark decays, retain all $t \bar{t}$ spin correlations and allow for arbitrary cuts on the final states. Therefore, one of the purposes of the present work is to fill this gap by extending the HELAC-NLO framework to include the full NWA. On one hand, having the possibility to provide theoretical predictions for both approaches within the same tool will facilitate systematic comparisons. On the other hand, such automation will open a new path for performing higher order calculations for more complex processes such as $t \bar{t} b \bar{b}, t \bar{t} j j$ or $t \bar{t} t \bar{t}$, for which only predictions with stable top quarks are available at NLO in QCD, see e.g. refs. [21, 46-52]. For $t \bar{t} b \bar{b}$, there are also predictions with NLO + PS accuracy, where top quarks are decayed by PS programs [53-55].

\footnotetext{
${ }^{1}$ NLO QCD predictions for $t \bar{t} \gamma$ in the NWA are presented in [29]. For $t \bar{t} Z$ and $t \bar{t} j$ they are described respectively in [43] and [37,44]. Finally, for the $t \bar{t} W^{ \pm}$process they are given in [45]. In addition, we note that all these predictions have already been heavily used in various experimental analyses both at the TeVatron and the LHC.
} 


\subsection{Implementing the NWA in HELAC-NLO}

In the conventional implementation of the NWA, the amplitudes of the various production and decay subprocesses are computed separately and subsequently combined. In order to preserve the $t \bar{t}$ spin correlations between the production and decay stages a careful bookkeeping of all matrix elements corresponding to different polarisations of the resonant particles is necessary. We will refer to this strategy as the bottom-up approach. The combinatorial burden increases with the number of resonant particles and with the number of sequential decays.

In HelaC-Nlo we consider, however, a different strategy for the implementation of the NWA, which we will refer to as the top-down approach. We construct the fully decayed final state using the standard Dyson-Schwinger recursive algorithms $[56,57]$ but restrict the computation to double resonant topologies only, discarding single- and non-resonant ones. Furthermore, we introduce additional modifications to the fermionic propagator. In case of the resonant propagator formula (2.1) amounts to the following replacement

$$
\frac{\not p_{f}+m_{f}}{\left(p_{f}^{2}-m_{f}^{2}\right)+i m_{f} \Gamma_{f}} \longrightarrow\left(\not p_{f}+m_{f}\right) \sqrt{\frac{\pi}{m_{f} \Gamma_{f}}},
$$

while the on-shell Dirac delta, $\delta\left(p_{t}^{2}-m_{t}^{2}\right)$, is contained in the phase-space. ${ }^{2}$ For the nonresonant one we have instead

$$
\frac{\not p_{f}+m_{f}}{\left(p_{f}^{2}-m_{f}^{2}\right)+i m_{f} \Gamma_{f}} \longrightarrow \frac{\not p_{f}+m_{f}}{\left(p_{f}^{2}-m_{f}^{2}\right)} .
$$

The numerator in eq. (2.4) can be left unchanged since in the on-shell limit we can write

$$
\left(\not p_{f}+m_{f}\right)=\sum_{s= \pm} u\left(p_{f}, s\right) \bar{u}\left(p_{f}, s\right) .
$$

A similar modification can be introduced in the case of the $W$ gauge boson propagator. The main advantage of the top-down approach is that it reduces the number of contributions to be calculated, and thus improves bookkeeping issues. The main challenge of this approach lies in developing an efficient algorithm for selecting double resonant topologies.

We would like to note here, that both the bottom-up and the top-down approaches are of course equivalent and should provide the same final answer. Different MC programs have, however, distinct internal structures. Given the level of complexity of such programs the approach that requires the minimal amount of structural changes in the code is the one to be incorporated. Because HELAC-NLO is designed to efficiently obtain one-loop helicity amplitudes and calculate total cross sections at NLO in QCD for multi-particle processes in the SM the top-down approach is more natural and conceptually easier to implement. Furthermore, it allows us to exploit highly optimised recursion algorithms that have already been employed to provide NLO QCD results for $t \bar{t}, t \bar{t} j, t \bar{t} \gamma$ and $t \bar{t} Z$ processes with the complete top quark off-shell effects included [30, 58-62].

\footnotetext{
${ }^{2}$ While carefully examining eq. (2.4) it may seem that there is a mismatch of dimensions on both sides of eq. (2.4). This is, however, not the case since the Dirac delta function has been moved to the phase-space for the right hand side of the equation.
} 


\subsection{Virtual corrections}

From the point of view of the virtual corrections the implementation of the NWA into the HELAC-NLO framework does not introduce additional complications. Again the biggest challenge comprises the efficient selection of the topologies that correspond to factorisable one-loop contributions. Schematic representation of one-loop contributions to the $t \bar{t} \gamma$ production process in the NWA is shown in figure 2. For simplicity we first assume that only top quarks are treated in the NWA. Consequently, the following three contributions are only considered

$$
\begin{aligned}
& p p \rightarrow t \bar{t} \gamma \rightarrow\left(b e^{+} \nu_{e}\right)\left(\bar{b} \mu^{-} \bar{\nu}_{\mu}\right) \gamma, \\
& p p \rightarrow t \bar{t} \rightarrow\left(b e^{+} \nu_{e} \gamma\right)\left(\bar{b} \mu^{-} \bar{\nu}_{\mu}\right), \\
& p p \rightarrow t \bar{t} \rightarrow\left(b e^{+} \nu_{e}\right)\left(\bar{b} \mu^{-} \bar{\nu}_{\mu} \gamma\right),
\end{aligned}
$$

where the parenthesis denotes the resonant structure. The one-loop amplitudes for each of the three processes listed above contain corrections to both production and decays. In the case where also the $W$ gauge boson is treated in the NWA the list of the contributions to be considered increases and is given by

$$
\begin{aligned}
& p p \rightarrow t \bar{t} \gamma \rightarrow\left(b W^{+}\right)\left(\bar{b} W^{-}\right) \gamma \rightarrow\left[b\left(e^{+} \nu_{e}\right)\right]\left[\bar{b}\left(\mu^{-} \bar{\nu}_{\mu}\right)\right] \gamma \\
& p p \rightarrow t \bar{t} \rightarrow\left(b W^{+} \gamma\right)\left(\bar{b} W^{-}\right) \rightarrow\left[b\left(e^{+} \nu_{e}\right) \gamma\right]\left[\bar{b}\left(\mu^{-} \bar{\nu}_{\mu}\right)\right] \\
& p p \rightarrow t \bar{t} \rightarrow\left(b W^{+}\right)\left(\bar{b} W^{-} \gamma\right) \rightarrow\left[b\left(e^{+} \nu_{e}\right)\right]\left[\bar{b}\left(\mu^{-} \bar{\nu}_{\mu}\right) \gamma\right] \\
& p p \rightarrow t \bar{t} \rightarrow\left(b W^{+}\right)\left(\bar{b} W^{-}\right) \rightarrow\left[b\left(e^{+} \nu_{e} \gamma\right)\right]\left[\bar{b}\left(\mu^{-} \bar{\nu}_{\mu}\right)\right] \\
& p p \rightarrow t \bar{t} \rightarrow\left(b W^{+}\right)\left(\bar{b} W^{-}\right) \rightarrow\left[b\left(e^{+} \nu_{e}\right)\right]\left[\bar{b}\left(\mu^{-} \bar{\nu}_{\mu} \gamma\right)\right]
\end{aligned}
$$

The description outlined above is fully automated and can be used to tackle the calculation of more complicated processes for which the full NWA predictions have not yet been computed.

\subsection{Real corrections}

The cancellation of soft and collinear radiation contributions in the NWA is performed using the Catani-Seymour dipole subtraction scheme [63]. Specifically, the formulation presented in ref. [64] for massive quarks has been used with the extension to arbitrary helicity eigenstates of the external partons [32], as implemented in HELAC-DiPOLES. In order to deal with gluon radiation in the production and decays of the on-shell top quarks some modifications have been made that will be outlined briefly below. For example, in order to deal with gluon radiation in the decay stage of the $t \bar{t} \gamma$ production process we have implemented a modified version of the specialised subtraction procedure introduced in ref. [65] for single top quark production.

At the production level, gluon radiation off the top quarks generates extra soft divergences that need to be cancelled. This is in contrast to the full calculation where intermediate top quark propagators are not affected by infrared divergences. Thus, we include the 

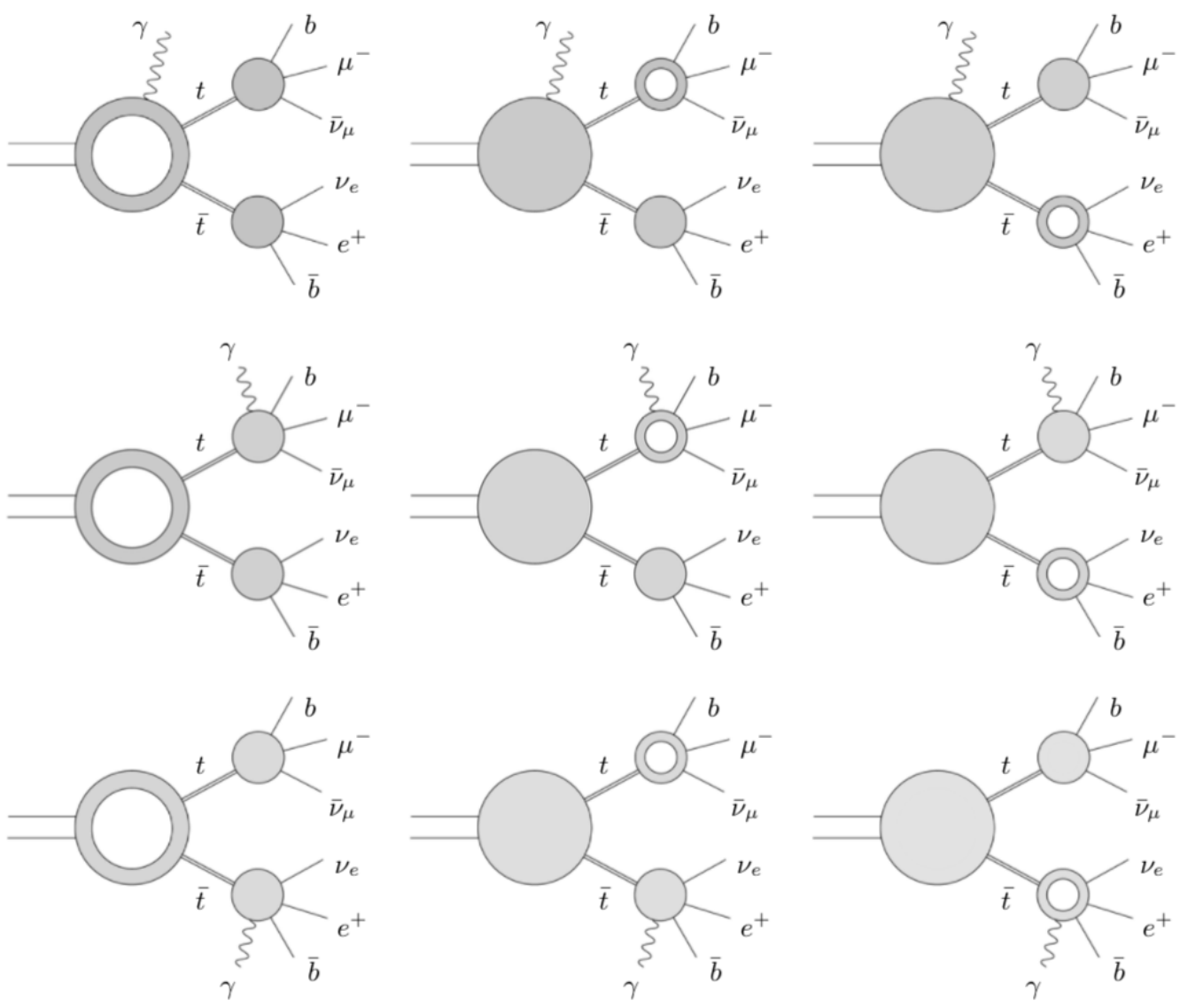

Figure 2. Schematic representation of one-loop contributions for the $t \bar{t} \gamma$ production process in the NWA. For simplicity only top quarks are treated in NWA. The three rows show the three contributions from eq. (2.7). The full blobs represent tree-level sub-amplitudes whereas the blobs with a hole denote sub-amplitudes with one-loop corrections included.

resonant top quarks into the list of emitters and compute the corresponding dipoles for the cases of final-state and initial-state spectators. The Catani-Seymour mapping is applied to the momentum entering the resonant propagator that we labelled as $p_{t}$. The latter is reconstructed from its decay products, see figure 3 for the graphical representation of the mapping. This mapping must then be carried out onto the decay products. Since the momenta of the decay products and the intermediate particle are subject to the mass-shell constraints, the new momenta can be obtained with a Lorentz transformation. This (ambiguous) Lorentz transformation is constructed as the product of two boosts, based on the observation that the momenta of the intermediate particle before and after the mapping are the same when expressed in their center-of-mass (CM) frames

$$
p_{t}^{\mathrm{CM}}=\tilde{p}_{t}^{\mathrm{CM}}=\left(m_{t}, \overrightarrow{0}\right) \text {. }
$$

Here $p_{t}^{\mathrm{CM}} \equiv \Lambda p_{t}$ and $\tilde{p}_{t}^{\mathrm{CM}} \equiv \tilde{\Lambda} \tilde{p}_{t}$ indicate the Lorentz transformations that bring the corresponding momenta into their CM frames and a tilde represents the mapped top quark 

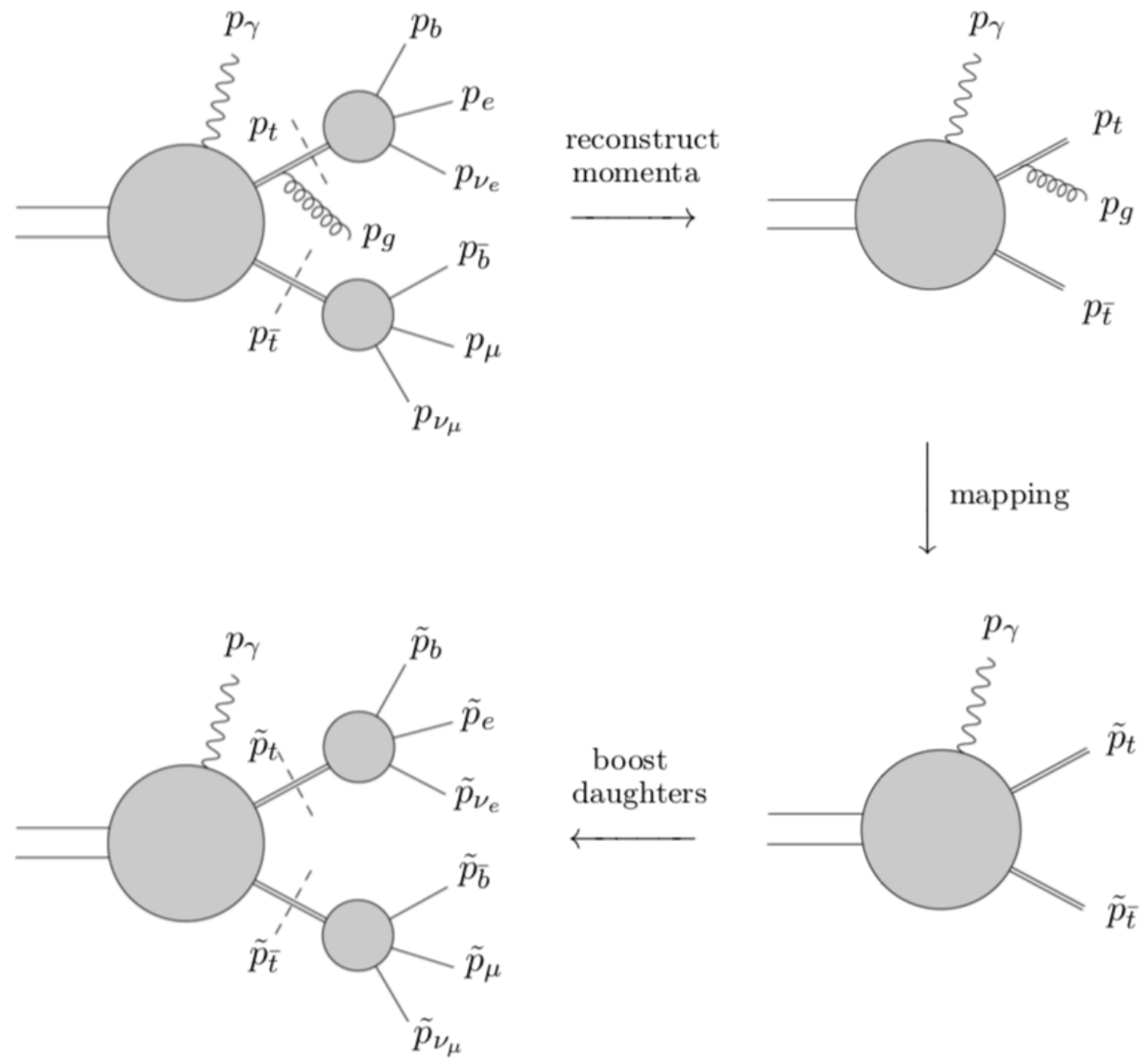

Figure 3. The phase-space mapping as applied to an intermediate emitter. The shown example refers to the case where the emitter is the top quark and the spectator is the anti-top quark.

momentum. From eq. (2.9) we obtain

$$
\tilde{p}_{t}=\left(\tilde{\Lambda}^{-1} \Lambda\right) p_{t} .
$$

This Lorentz transformation is applied again into the momenta of the decay products to construct the mapped final states. Even though the mapping is not conceptually different from the standard Catani-Seymour implementation, the form of the new subtraction terms is not so trivial. Because the top quark propagator is already summed up over its polarisation states it seems that one cannot just use the polarised formulae from ref. [32]. The divergence, however, has a pure soft nature, so it is independent of the top quark polarisations. Consequently, standard, non-polarised Catani-Seymour dipole can be used with an additional symmetry factor of $1 / 2$ which compensates for the summation over the two polarisations of the gluon.

In the following we shall shortly discuss the treatment of gluon radiation in top quark decays. The Catani-Seymour subtraction scheme can be extended straightforwardly to top quark decays. In ref. [65] such extension, that preserves the momentum of the decaying particle and it is therefore applicable to the case of top quark decays, has been proposed. 
It has been later extended in ref. [29] to the case of radiative decays. We employ the scheme of ref. [65] to construct subtraction terms for the final-initial case where initial state here means the decaying top quark. The latter are available in the literature only in the unpolarised form. Thus, we have derived the extension of the subtraction term of ref. [65] to the polarised case which, for massless $b$ quarks, reads

$$
\begin{aligned}
& D\left(\left(p_{t}+p_{g}\right)^{2},\left(p_{b}+p_{g}\right)^{2}, m_{t}^{2}\right)_{\lambda \lambda^{\prime} \lambda_{b} \lambda_{g}} \\
& \quad=g^{2} \mu^{2 \epsilon} C_{F}\left[\frac{1}{p_{b} \cdot p_{g}}\left(\frac{z^{2}}{(1-z)}+\delta_{\lambda_{b} \lambda_{g}}(1+z)\right)-\frac{1}{2} \frac{m_{t}^{2}}{\left(p_{t} \cdot p_{g}\right)^{2}}\right] \delta_{\lambda \lambda_{b}} \delta_{\lambda \lambda^{\prime}} .
\end{aligned}
$$

Here $\lambda_{b}, \lambda_{g}$ are the helicity eigenstates of the external $b$-quark and gluon respectively and $\lambda, \lambda^{\prime}$ are the helicity eigenstates that enter the Born matrix element. The rest of the notation follows the original reference. We emphasise that the modifications to the subtraction terms described above do not affect the analytical structure of the integrated dipoles. We used the formulae that were already available in the literature without making any additional changes. We note here that all other cases have been addressed with the standard implementation of the Catani-Seymour subtraction scheme that has already been available in the HelaC-Dipoles software.

\section{$2.4 \quad$ Numerical checks}

In order to test our implementation of the NWA in the HELAC-NLO system we have performed a number of cross-checks. First of all, the implementation of the real corrections has been validated for the $p p \rightarrow t \bar{t} \gamma$ process in the di-lepton channel by checking that the subtraction terms match the singular behaviour of the matrix elements for increasingly collinear and soft limits. We have explicitly checked the cancellation of the $\epsilon$ poles coming from loop contributions against those of the integrated subtraction terms for a few phase space points. Additionally, we have verified that our predictions for the real emission part do not depend on the particular value of the $\alpha_{\max }$ parameter. The parameter, which controls the size of the subtraction region, has been first proposed for the Catani-Seymour subtraction scheme in refs. [66, 67]. Furthermore, we have performed extensive checks of the NWA version of the HELAC-NLO framework against other publicly available calculations. The only publicly available results for the $t \bar{t} \gamma$ production process in full NWA comprise the lepton plus jets top quark decay channel [29]. Thus, we cannot compare to them directly at the moment. Instead, we focused on the simpler process, i.e the $t \bar{t}$ production process with full leptonic decays of the top quarks. As a first step we have checked that our results for the fully inclusive NLO cross section agree with the NLO part of the calculation of ref. [39]. On a more exclusive ground, we have reproduced the NLO differential cross section distributions that are presented in refs. [41, 42]. Finally, we have cross-checked our results with NLO QCD corrections included separately for the production and the decay stages against the results obtained with the public MC program MCFM [38]. 


\section{$3 \quad$ LHC setup}

We study $p p \rightarrow e^{+} \nu_{e} \mu^{-} \bar{\nu}_{\mu} b \bar{b} \gamma+X$ for the LHC Run II energy of $\sqrt{s}=13 \mathrm{TeV}$ at $\mathcal{O}\left(\alpha_{s}^{3} \alpha^{5}\right)$. Our calculation uses the following parton distribution functions (PDFs): CT14 (the default PDF set) [68], NNPDF3.0 [69] and MMHT14 [70]. The number of active flavours is set to $N_{F}=5$, however, contributions induced by the bottom-quark parton density are neglected due to their numerical insignificance. We employ the following SM parameters

$$
\begin{aligned}
m_{W} & =80.385 \mathrm{GeV}, & \Gamma_{W} & =2.0988 \mathrm{GeV}, \\
m_{Z} & =91.1876 \mathrm{GeV}, & \Gamma_{Z} & =2.50782 \mathrm{GeV}, \\
G_{\mu} & =1.166378 \times 10^{-5} \mathrm{GeV}^{-2}, & \sin ^{2} \theta_{W} & =1-m_{W}^{2} / m_{Z}^{2} .
\end{aligned}
$$

Since leptonic $W$ gauge boson decays do not receive NLO QCD corrections, to account for some higher order effects the NLO QCD values for the gauge boson widths are used everywhere, i.e. for LO and NLO matrix elements. The electroweak coupling is derived from the Fermi constant $G_{\mu}$ according to

$$
\alpha=\frac{\sqrt{2} G_{\mu} m_{W}^{2} \sin ^{2} \theta_{W}}{\pi} .
$$

For the emission of the isolated photon, however, $\alpha_{\mathrm{QED}}=1 / 137$ is used instead. The top quark mass is set to $m_{t}=173.2 \mathrm{GeV}$. All other QCD partons including $b$ quarks as well as leptons are treated as massless. The final state jets are constructed from the final state partons $(j)$ with pseudo-rapidity $|\eta(j)|<5$ via the IR-safe anti- $k_{T}$ jet algorithm [71] with $R=0.4$. We require at least two jets for our process, of which exactly two must be bottom flavoured jets. We impose the following cuts on the transverse momenta and the rapidity of two recombined $b$-jets, which we assume to be always tagged

$$
p_{T}(b)>40 \mathrm{GeV}, \quad|y(b)|<2.5, \quad \Delta R(b b)>0.4 .
$$

The last cut, i.e. the separation between the $b$-jets, is implied by the jet algorithm. Furthermore, we request two charged leptons, missing transverse momentum and an isolated hard photon. The latter is defined with $p_{T}(\gamma)>25 \mathrm{GeV}$ and $|y(\gamma)|<2.5$. To avoid QED collinear singularities from photon emission that are introduced by the $q \rightarrow q \gamma$ splitting, a separation between quark and photon is needed. This in turn introduces a separation between photons and gluons as all partons are treated in the same way. Therefore, for a given $p_{T}(\gamma)$ an angular restriction on the soft gluon emission is introduced compromising the cancelation of infrared divergences. To ensure infrared safety we use the Frixione photon isolation prescription described in ref. [72], which is based on a modified cone approach. The (smooth) photon isolation condition is implemented in the same way for quarks and gluons. Specifically, we reject the event unless the following condition is fulfilled

$$
\sum_{i} E_{T}(i) \Theta(R-R(\gamma i)) \leq \epsilon_{\gamma} E_{T}(\gamma)\left(\frac{1-\cos (R)}{1-\cos (R(\gamma j))}\right)^{n} \quad \forall R \leq R(\gamma j),
$$


where $E_{T}(i)$ stands for the transverse energy of the parton $i$ and $E_{T}(\gamma)$ is the transverse energy of the photon. Because of the $\Theta(R-R(\gamma i))$ condition the sum gets contributions only when the angular distance of the parton $i$ from the photon is less than or equal to $R$. We set the following values for the parameters of the isolation criterion $\epsilon_{\gamma}=1, n=1$ and $R(\gamma j)=0.4$. Finally, $R(\gamma i)$ is given by

$$
R(\gamma i)=\sqrt{(y(\gamma)-y(i))^{2}+(\varphi(\gamma)-\varphi(i))^{2}}
$$

where $y$ and $\varphi$ are the rapidity and azimuthal angle respectively. In eq. (3.3) the $(1-\cos (R))$ term suppresses the collinear singularities, which are present for $R \rightarrow 0$. On the other hand, arbitrarily soft radiation is allowed inside the $R(\gamma j)$ cone preserving the cancellation of infrared poles in the calculation. Basic selection cuts are applied to charged leptons to ensure that they are observed inside the detector and well separated from each other

$$
p_{T}(\ell)>30 \mathrm{GeV}, \quad \Delta R(\ell \ell)>0.4, \quad|y(\ell)|<2.5,
$$

where $\ell=e^{+}, \mu^{-}$. Moreover, we impose that charged leptons are well separated from the isolated photon and from $b$-jets

$$
\Delta R(\ell b)>0.4, \quad \Delta R(\ell \gamma)>0.4, \quad \Delta R(b \gamma)>0.4 .
$$

We additionally place a requirement on the missing transverse momentum $p_{T}^{\text {miss }}>20 \mathrm{GeV}$. Finally, we impose no restrictions on the kinematics of the extra (light) jet other than it should be separated from the isolated photon. In this work we have utilised two different forms for the factorisation and renormalisation scales for the results in the NWA: $\mu_{0}=$ $\mu_{R}=\mu_{F}=m_{t} / 2$ and $\mu_{0}=\mu_{R}=\mu_{F}=H_{T} / 4$. We show theoretical predictions for the NWA with the fixed scale choice mostly for comparison. We note here, however, that the fixed scale choice is still commonly used in various phenomenological studies. In the case of full off-shell results, on the other hand, only the dynamical scale choice, $\mu_{0}=H_{T} / 4$, is used since this is our recommended scale choice for the process under consideration, see refs. $[15,30]$. The total transverse momentum of the system, $H_{T}$, is defined according to

$$
H_{T}=p_{T}\left(e^{+}\right)+p_{T}\left(\mu^{-}\right)+p_{T}^{m i s s}+p_{T}\left(b_{1}\right)+p_{T}\left(b_{2}\right)+p_{T}(\gamma)
$$

where $p_{T}\left(b_{1}\right)$ and $p_{T}\left(b_{2}\right)$ are bottom-flavoured jets and $p_{T}^{\text {miss }}$ is the missing transverse momentum from the two neutrinos. Even though we assume that the central scale $\mu_{R}=\mu_{F}=$ $\mu_{0}$ is the same for both the renormalisation and factorisation scales the scale systematics is evaluated by varying $\mu_{R}$ and $\mu_{F}$ independently. Specifically, we vary them in the following range

$$
\frac{1}{2} \mu_{0} \leq \mu_{R}, \mu_{F} \leq 2 \mu_{0}, \quad \frac{1}{2} \leq \frac{\mu_{R}}{\mu_{F}} \leq 2 .
$$

The top quark width, as calculated from [73-75], is taken to be

$$
\begin{aligned}
\Gamma_{t, \mathrm{off}-\text { shell }}^{\mathrm{LO}} & =1.47848 \mathrm{GeV}, & \Gamma_{t, \mathrm{off}-\text { shell }}^{\mathrm{NLO}} & =1.35159 \mathrm{GeV}, \\
\Gamma_{t, \mathrm{NWA}}^{\mathrm{LO}} & =1.50176 \mathrm{GeV}, & \Gamma_{t, \mathrm{NWA}}^{\mathrm{NLO}} & =1.37289 \mathrm{GeV} .
\end{aligned}
$$




\begin{tabular}{|c|c|c|c|c|c|c|c|}
\hline PDF & $p_{T}(b)$ & $\sigma^{\mathrm{LO}}[\mathrm{fb}]$ & $\delta_{\text {scale }}$ & $\sigma^{\mathrm{NLO}}[\mathrm{fb}]$ & $\delta_{\text {scale }}$ & $\delta_{\mathrm{PDF}}$ & $\mathcal{K}$ \\
\hline \multirow[t]{4}{*}{ CT14 } & 25 & 10.68 & $\begin{array}{l}+3.54(33 \%) \\
-2.49(23 \%)\end{array}$ & 11.19 & $\begin{array}{l}+0.16(1 \%) \\
-0.54(5 \%)\end{array}$ & $\begin{array}{l}+0.32(3 \%) \\
-0.35(3 \%)\end{array}$ & 1.05 \\
\hline & 30 & 9.58 & $\begin{array}{l}+3.18(33 \%) \\
-2.24(23 \%)\end{array}$ & 9.93 & $\begin{array}{l}+0.14(1 \%) \\
-0.54(5 \%)\end{array}$ & $\begin{array}{l}+0.28(3 \%) \\
-0.31(3 \%)\end{array}$ & 1.04 \\
\hline & 35 & 8.44 & $\begin{array}{l}+2.80(33 \%) \\
-1.97(23 \%)\end{array}$ & 8.69 & $\begin{array}{l}+0.12(1 \%) \\
-0.50(6 \%)\end{array}$ & $\begin{array}{l}+0.25(3 \%) \\
-0.27(3 \%)\end{array}$ & 1.03 \\
\hline & 40 & 7.32 & $\begin{array}{l}+2.45(33 \%) \\
-1.71(23 \%)\end{array}$ & 7.50 & $\begin{array}{l}+0.11(1 \%) \\
-0.45(6 \%)\end{array}$ & $\begin{array}{l}+0.22(3 \%) \\
-0.23(3 \%)\end{array}$ & 1.02 \\
\hline \multirow[t]{4}{*}{ ММHТ14 } & 25 & 11.59 & $\begin{array}{l}+4.22(36 \%) \\
-2.88(25 \%)\end{array}$ & 11.29 & $\begin{array}{l}+0.16(1 \%) \\
-0.57(5 \%)\end{array}$ & $\begin{array}{l}+0.24(2 \%) \\
-0.22(2 \%)\end{array}$ & 0.97 \\
\hline & 30 & 10.38 & $\begin{array}{l}+3.78(36 \%) \\
-2.58(25 \%)\end{array}$ & 10.02 & $\begin{array}{l}+0.13(1 \%) \\
-0.58(6 \%)\end{array}$ & $\begin{array}{l}+0.22(2 \%) \\
-0.19(2 \%)\end{array}$ & 0.97 \\
\hline & 35 & 9.12 & $\begin{array}{l}+3.33(36 \%) \\
-2.26(25 \%)\end{array}$ & 8.77 & $\begin{array}{l}+0.11(1 \%) \\
-0.54(6 \%)\end{array}$ & $\begin{array}{l}+0.19(2 \%) \\
-0.17(2 \%)\end{array}$ & 0.96 \\
\hline & 40 & 7.90 & $\begin{array}{l}+2.89(37 \%) \\
-1.96(25 \%)\end{array}$ & 7.57 & $\begin{array}{l}+0.09(1 \%) \\
-0.48(6 \%)\end{array}$ & $\begin{array}{l}+0.16(2 \%) \\
-0.15(2 \%)\end{array}$ & 0.96 \\
\hline \multirow[t]{4}{*}{ NNPDF3.0 } & 25 & 10.78 & $\begin{array}{l}+3.82(35 \%) \\
-2.62(24 \%)\end{array}$ & 11.62 & $\begin{array}{l}+0.17(1 \%) \\
-0.58(5 \%)\end{array}$ & $\begin{array}{l}+0.16(1 \%) \\
-0.16(1 \%)\end{array}$ & 1.08 \\
\hline & 30 & 9.65 & $\begin{array}{l}+3.42(35 \%) \\
-2.34(24 \%)\end{array}$ & 10.31 & $\begin{array}{l}+0.14(1 \%) \\
-0.58(6 \%)\end{array}$ & $\begin{array}{l}+0.14(1 \%) \\
-0.14(1 \%)\end{array}$ & 1.07 \\
\hline & 35 & 8.48 & $\begin{array}{l}+3.01(35 \%) \\
-2.05(24 \%)\end{array}$ & 9.02 & $\begin{array}{l}+0.12(1 \%) \\
-0.53(6 \%)\end{array}$ & $\begin{array}{l}+0.12(1 \%) \\
-0.12(1 \%)\end{array}$ & 1.06 \\
\hline & 40 & 7.34 & $\begin{array}{l}+2.61(36 \%) \\
-1.78(24 \%)\end{array}$ & 7.79 & $\begin{array}{l}+0.10(1 \%) \\
-0.48(6 \%)\end{array}$ & $\begin{array}{l}+0.11(1 \%) \\
-0.11(1 \%)\end{array}$ & 1.06 \\
\hline
\end{tabular}

Table 1. Integrated cross sections for the $p p \rightarrow e^{+} \nu_{e} \mu^{-} \bar{\nu}_{\mu} b \bar{b} \gamma+X$ production process at the LHC with $\sqrt{s}=13 \mathrm{TeV}$. Results are evaluated using $\mu_{R}=\mu_{F}=\mu_{0}=H_{T} / 4$ for three different PDF sets and four different $p_{T}(b)$ cuts for the $b$-jets. Also given are theoretical uncertainties coming from scale variation, $\delta_{\text {scale, }}$, and from PDFs, $\delta_{P D F}$. In the last column a $\mathcal{K}$-factor, that is defined as $\mathcal{K}=\sigma^{\mathrm{NLO}} / \sigma^{\mathrm{LO}}$, is shown.

The value of $\alpha_{s}$ used for the top quark width $\Gamma_{t}^{\mathrm{NLO}}$ calculation is taken at $\alpha_{s}\left(m_{t}\right)$. This $\alpha_{s}$ is independent of $\alpha_{s}\left(\mu_{0}\right)$ that goes into the matrix element and PDF calculations. The latter is used to describe the dynamics of the whole process, while the former only the top quark decays. For more details we refer the reader to our previous publications [15, 30].

\section{Stability of the full off-shell result}

Before comparing various approaches for the top quark decay modelling for the $p p \rightarrow$ $e^{+} \nu_{e} \mu^{-} \bar{\nu}_{\mu} b \bar{b} \gamma$ process, we first investigate the stability of the full off-shell results. Because they constitute the most realistic NLO computation for top quark pair production with an isolated photon in hadronic collision in the di-lepton top quark decay channel we would like to analyse them more precisely. In table 1 we present LO and NLO QCD predictions for the integrated cross sections for three PDF sets and for different values of the cut on the transverse momentum of the $b$-jet. We vary the cut in the range of $25-40 \mathrm{GeV}$ in steps of $5 \mathrm{GeV}$. The values of $\sigma^{\mathrm{LO}}$ and $\sigma^{\mathrm{NLO}}$ are evaluated using $\mu_{0}=H_{T} / 4$. Theoretical 
uncertainties coming from scale variation are denoted by $\delta_{\text {scale }}$ and from PDFs as $\delta_{\text {PDF }}$. Finally, in the last column the $\mathcal{K}$-factor, defined as $\mathcal{K}=\sigma^{\mathrm{NLO}} / \sigma^{\mathrm{LO}}$, is shown. The scale dependence is derived with the standard seven-point variation around the central value of the scale $\mu_{0}$ and indicated by the upper and lower indices. They correspond to the minimum and maximum of the resulting cross sections. For the PDF uncertainties we use the corresponding prescription from each group to provide the $68 \%$ confidence level (C.L.) PDF uncertainties. Both CT14 PDFs and MMHT14 PDFs include a central set and error sets in the Hessian representation. In that case we use the asymmetric expression for PDF uncertainties. Additionally, the CT14 errors are rescaled by a factor 1/1.645 since they are provided at 90\% C.L. On the other hand, for the NNPDF3.0 PDF sets PDF uncertainties are obtained using the replicas method.

We can observe that the scale uncertainty is reduced considerably through the inclusion of the NLO QCD corrections. PDF uncertainties are of the order of a few \%. These findings do not take into account additional systematics coming from the underlying assumptions that enter the parametrisation of different PDF sets. They simply cannot be quantified within a given scheme. We therefore additionally present results for other PDF sets. All three are recommended to be used for applications at the LHC Run II [76]. We see that CT14, MMHT14 and NNPDF3.0 NLO results differ at most by $4 \%$, which is comparable to the individual estimates of PDF systematics. Overall, the PDF uncertainties for the process under scrutiny are below the theoretical uncertainties due to the scale dependence, which remain the dominant source of the theoretical systematics.

The most important message here, however, is that the above findings for $\delta_{\text {scale }}$ are rather insensitive to the chosen $p_{T}(b)$ cut value. We could uncover only variations at the percent level. In particular, there is no big difference for the lowest cut value of the $p_{T}(b)$ cut, $25 \mathrm{GeV}$, and the value we use as the default one in our analysis, i.e. $40 \mathrm{GeV}$. This suggests that the perturbative expansion is not spoiled by the appearance of large logarithms, thus, under excellent theoretical control. Finally, even though NLO QCD corrections for different PDF sets and for all values of the $p_{T}(b)$ cut are varying between positive or negative ones, they are all consistently below $10 \%$.

Having established stability of the full off-shell results with respect to the $p_{T}(b)$ cut we move on to the main part of the paper and investigate differences between full off-shell results and the calculations in the NWA.

\section{Phenomenological results for integrated cross sections}

In the following we compare the full off-shell results with the calculations in the NWA. In the latter case two versions will be examined: the full NWA and the NWA LOdecay (NWA with LO decays of top quarks and photon radiation in the production stage only). Theoretical predictions for these three cases, that have been evaluated for the choice of the kinematic cuts and SM parameters as described in the previous section, are listed in table 2. We additionally provide theoretical uncertainties from the scale dependence. Moreover, all results are evaluated with the CT14 PDF sets. We note here, that results for top quark pair production and also for $t \bar{t}$ process with an additional jet or the gauge boson in the full 


\begin{tabular}{|lcc|}
\hline Modelling ApPROACH & $\sigma^{\mathrm{LO}}[\mathrm{fb}]$ & $\sigma^{\mathrm{NLO}}[\mathrm{fb}]$ \\
\hline full off-shell $\left(\mu_{0}=H_{T} / 4\right)$ & $7.32_{-1.71(23 \%)}^{+2.45(33 \%)}$ & $7.50_{-0.45(6 \%)}^{+0.11(1 \%)}$ \\
\hline NWA $\left(\mu_{0}=m_{t} / 2\right)$ & $8.08_{-1.96(24 \%)}^{+2.84(35 \%)}$ & $7.28_{-0.03(0.4 \%)}^{-0.99(13 \%)}$ \\
NWA $\left(\mu_{0}=H_{T} / 4\right)$ & $7.18_{-1.68(23 \%)}^{+2.39(33 \%)}$ & $7.33_{-0.24(3.3 \%)}^{-0.43(5.9 \%)}$ \\
\hline NWA $_{\gamma-\text { prod }}\left(\mu_{0}=m_{t} / 2\right)$ & $4.52_{-1.11(24 \%)}^{+1.63(36 \%)}$ & $4.13_{-0.05(1.2 \%)}^{-0.53(13 \%)}$ \\
NWA $_{\gamma-\text { prod }}\left(\mu_{0}=H_{T} / 4\right)$ & $3.85_{-0.90(23 \%)}^{+1.29(33 \%)}$ & $4.15_{-0.21(5.1 \%)}^{-0.12(2.3 \%)}$ \\
\hline NWA $_{\gamma-\text { decay }}\left(\mu_{0}=m_{t} / 2\right)$ & $3.56_{-0.85(24 \%)}^{+1.20(34 \%)}$ & $3.15_{+0.03(0.9 \%)}^{-0.46(15 \%)}$ \\
NWA $_{\gamma-\text { decay }}\left(\mu_{0}=H_{T} / 4\right)$ & $3.33_{-0.77(23 \%)}^{+1.10(33 \%)}$ & $3.18_{-0.03(0.9 \%)}^{-0.31(9.7 \%)}$ \\
\hline NWA $_{\text {LOdecay }}\left(\mu_{0}=m_{t} / 2\right)$ & & $4.85_{-0.48(5.9 \%)}^{+0.26(5.4 \%)}$ \\
NWA $_{\text {LOdecay }}\left(\mu_{0}=H_{T} / 4\right)$ & & $4.63_{-0.52(11 \%)}^{+0.44(9.5 \%)}$ \\
\hline
\end{tabular}

Table 2. Integrated cross sections for the $p p \rightarrow e^{+} \nu_{e} \mu^{-} \bar{\nu}_{\mu} b \bar{b} \gamma+X$ production process at the LHC with $\sqrt{s}=13 \mathrm{TeV}$. Results for various approaches for the modelling of top quark decays are listed. Also given is the full off-shell result. We additionally provide theoretical uncertainties as obtained from the scale dependence. The CT14 PDF set is employed.

NWA are usually provided as a consistent expansion in $\alpha_{s}$. More explicitly, the NLO top quark decay width, that appears as $\Gamma_{t, \mathrm{NLO}}^{-2}$ and is a part of the branching fractions for the corresponding $t(\bar{t})$ decay, has usually been expanded in powers of $\alpha_{s}$. For the comparison at hand, however, such expansion has not been performed and $\Gamma_{t, \mathrm{NLO}}^{-2}$ in the theoretical prediction in the NWA is valid to all orders in the strong coupling constant. The reason for not using this expansion for the results in the NWA should be clear, namely such a procedure can not be directly applied to the full off-shell calculations. Because the main purpose of the paper is a consistent comparison between the NWA and the full off-shell results such approach seems to be more appropriate. Nevertheless, we have checked that the difference between the expanded and unexpanded full NWA results is at the level of $1 \%$ independently of the scale choice.

We first assess the size of the non-factorisable corrections for our setup. Finite top quark width effects change the NLO cross section by less than $3 \%$ independent of the scale choice. This finding is consistent with the expected uncertainty of the NWA that is of the order of $\mathcal{O}\left(\Gamma_{t} / m_{t}\right) \approx 0.8 \%$. At LO we have received $2 \%$ corrections for $\mu_{0}=H_{T} / 4$ and $10 \%$ for $\mu_{0}=m_{t} / 2$. We note, however, that should we compare the LO NWA result with $\mu_{0}=m_{t} / 2$ to the full off-shell one with the same scale choice we would also get only $2 \%$ corrections. We would like to add at this point, that in ref. [30] the size of the top quark non-factorisable corrections has been estimated for the $p p \rightarrow e^{+} \nu_{e} \mu^{-} \bar{\nu}_{\mu} b \bar{b} \gamma$ process from the full off-shell result by rescaling the coupling of the top quark to the $W$ boson and the 
$b$ quark as well as the coupling of $W$ and the leptons by several large factors, as described in ref. [58]. This approach should mimic the $\Gamma_{t} \rightarrow 0$ limit when the scattering cross section factorises into on-shell production and decay. Indeed, using this method we reported $1.5 \%$ for LO and $2.5 \%$ for NLO with $\mu_{0}$ set to $m_{t} / 2$. Our current findings confirm that rescaling works very accurately for the process at hand where rather inclusive cuts on the final states have been applied.

In table 2 we additionally quote results for the LO and NLO QCD cross sections where photon radiation occurs either in the production $(p p \rightarrow t \bar{t} \gamma)$ or in the decay stage $\left(t \rightarrow b W^{+} \gamma \rightarrow b e^{+} \nu_{e} \gamma, t \rightarrow b W^{+} \rightarrow b e^{+} \nu_{e} \gamma, \bar{t} \rightarrow \bar{b} W^{-} \gamma \rightarrow \bar{b} \mu^{-} \bar{\nu}_{\mu} \gamma, \bar{t} \rightarrow \bar{b} W^{-} \rightarrow \bar{b} \mu^{-} \bar{\nu}_{\mu} \gamma\right)$ processes. In this way we can estimate the importance of the photon emission in top quark decays. Using results from table 2, we have calculated that at NLO more than $57 \%$ of photons are emitted either from the initial state light quarks or off-shell top quarks that afterwards go on-shell. ${ }^{3}$ This means that $43 \%$ of all isolated photons are emitted in the decay stage, i.e. either from on-shell top quarks or its decay products ( $b$-jets, $W$ gauge bosons and/or charged leptons). This conclusion is independent of the scale choice. Similar estimates can be obtained at LO. Consequently, the radiative decay of the top quark must be incorporated into theoretical predictions for $t \bar{t} \gamma$ production at the LHC since it yields a significant contribution to the cross section. Once the $p_{T}(b)$ cut is lowered to $25 \mathrm{GeV}$, i.e. to the value that is currently used in measurements of inclusive and differential crosssections of $t \bar{t} \gamma$ production in the $e \mu$ channel at $13 \mathrm{TeV}$ by the ATLAS collaboration [6], the photon contribution in the decay stage increases up to almost 50\%. This means that photon radiation is distributed evenly between the $t \bar{t} \gamma$ production process and the leptonic top quark decay stages.

Table 2 also shows results for the special case of the NWA, i.e. for the $\mathrm{NWA}_{\text {LOdecay }}$. They comprise NLO QCD corrections to the production of $t \bar{t} \gamma$ and LO top quark decays. Furthermore, photon radiation is restricted only to the production stage. Such a prediction should mimic the computation of ref. [23] where the NLO QCD corrections are computed for the $t \bar{t} \gamma$ production stage but include neither exact LO spin correlations nor radiative corrections to decays. On top of it, ref. [23] omits photon emission in the parton shower evolution. Because the contribution from photon emission in top quark decays is large and NLO QCD corrections to decays are also relevant it is not surprising that NWA LOdecay result can not reproduce the correct normalisation. The discrepancy to the NWA approach amounts to $50 \%(58 \%)$ for $\mu_{0}=m_{t} / 2\left(\mu_{0}=H_{T} / 4\right)$. NLO QCD corrections to the top quark decays are negative and at the level of $17 \%(12 \%)$ when $\mu_{0}=m_{t} / 2\left(\mu_{0}=H_{T} / 4\right)$ is employed in the NWA.

Finally, in table 2 theoretical uncertainties as obtained from the scale dependence are provided for all cases that we have considered up until now. When comparing the full offshell case with the full NWA one at NLO in QCD we observe that theoretical uncertainties are not underestimated when the NWA is employed. Instead, they are consistent at the level of $6 \%$ for $\mu_{0}=H_{T} / 4$ and $13 \%$ for $\mu_{0}=m_{t} / 2$, see ref. [30] for the full off-shell results at NLO in QCD with the fixed scale choice.

\footnotetext{
${ }^{3}$ At the central value of the scale $g g$ channel dominates the total LO $p p \rightarrow e^{+} \nu_{e} \mu^{-} \bar{\nu}_{\mu} b \bar{b} \gamma$ cross section by $79 \%$ and it is followed by the $q \bar{q}+\bar{q} q$ channel with $21 \%$.
} 

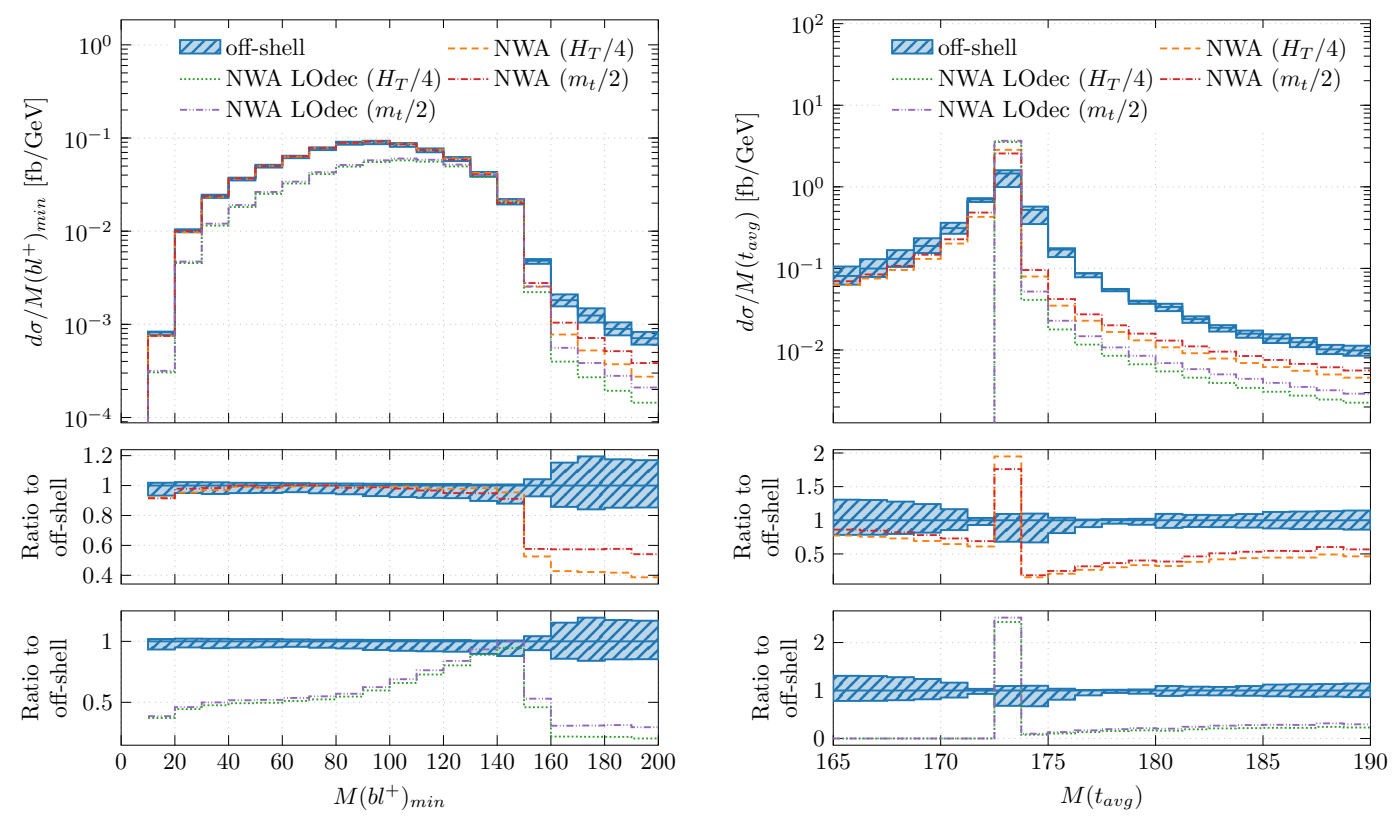

Figure 4. Differential cross section distribution as a function of the minimum invariant mass of the positron and bottom-jet, $M\left(b e^{+}\right)_{\min }$, and the (averaged) invariant mass of the top quark, $M\left(t_{\mathrm{avg}}\right)$, for the $p p \rightarrow e^{+} \nu_{e} \mu^{-} \bar{\nu}_{\mu} b \bar{b} \gamma$ process at the LHC run II with $\sqrt{s}=13 \mathrm{TeV}$. The CT14 PDF set is employed.

\section{Phenomenological results for differential cross sections}

\subsection{Off-shell vs. on-shell top quark modelling}

In the following we examine the size of top quark off-shell effects at the differential level. To this end we compare differential cross sections for a few observables at NLO in QCD using three different theoretical descriptions: the NWA, the NWA $\mathrm{LOdecay}_{\text {and }}$ results with the full off-shell effects. In the case of NWA, two scale choices, $\mu_{0}=m_{t} / 2$ and $\mu_{0}=H_{T} / 4$, are used, whereas for the full off-shell case only the latter is utilised. We show theoretical uncertainties as obtained from the scale dependence for the full off-shell case since we are only interested in effects that exceed the theoretical uncertainties. For all observables we employed the CT14 PDF set. The upper plots will show absolute NLO QCD predictions for three different theoretical descriptions. The ratios to the full off-shell result including its scale uncertainty band will be plotted in the middle and bottom plots.

In figure 4 we start with the two observables that are very well known from the top quark mass measurement in the $t \bar{t}(j)$ production process, see e.g. refs. [61, 77-79]. Specifically, we plot the minimum invariant mass of the positron and $b$-jet, $M\left(b e^{+}\right)_{\min }$, and the (averaged) invariant mass of the top quark, $M\left(t_{\text {avg }}\right)$. Because generally one can not distinguish which $b$-jet should be paired with the positron we define the $M\left(b e^{+}\right)_{\text {min }}$ observable as $M\left(b e^{+}\right)_{\min }=\min \left\{M\left(b_{1} e^{+}\right), M\left(b_{2} e^{+}\right)\right\}$, where $b_{1}$ and $b_{2}$ are bottom jets. Such criterion selects the correct pairing in approximately $85 \%$ of the cases [80]. At lowest order in perturbative expansion when both top quarks and $W$ gauge bosons are treated as on-shell particles there is a strict kinematical limit for $M\left(b e^{+}\right)$given by $M\left(b e^{+}\right)=\sqrt{m_{t}^{2}-m_{W}^{2}} \approx 153 \mathrm{GeV}$. 

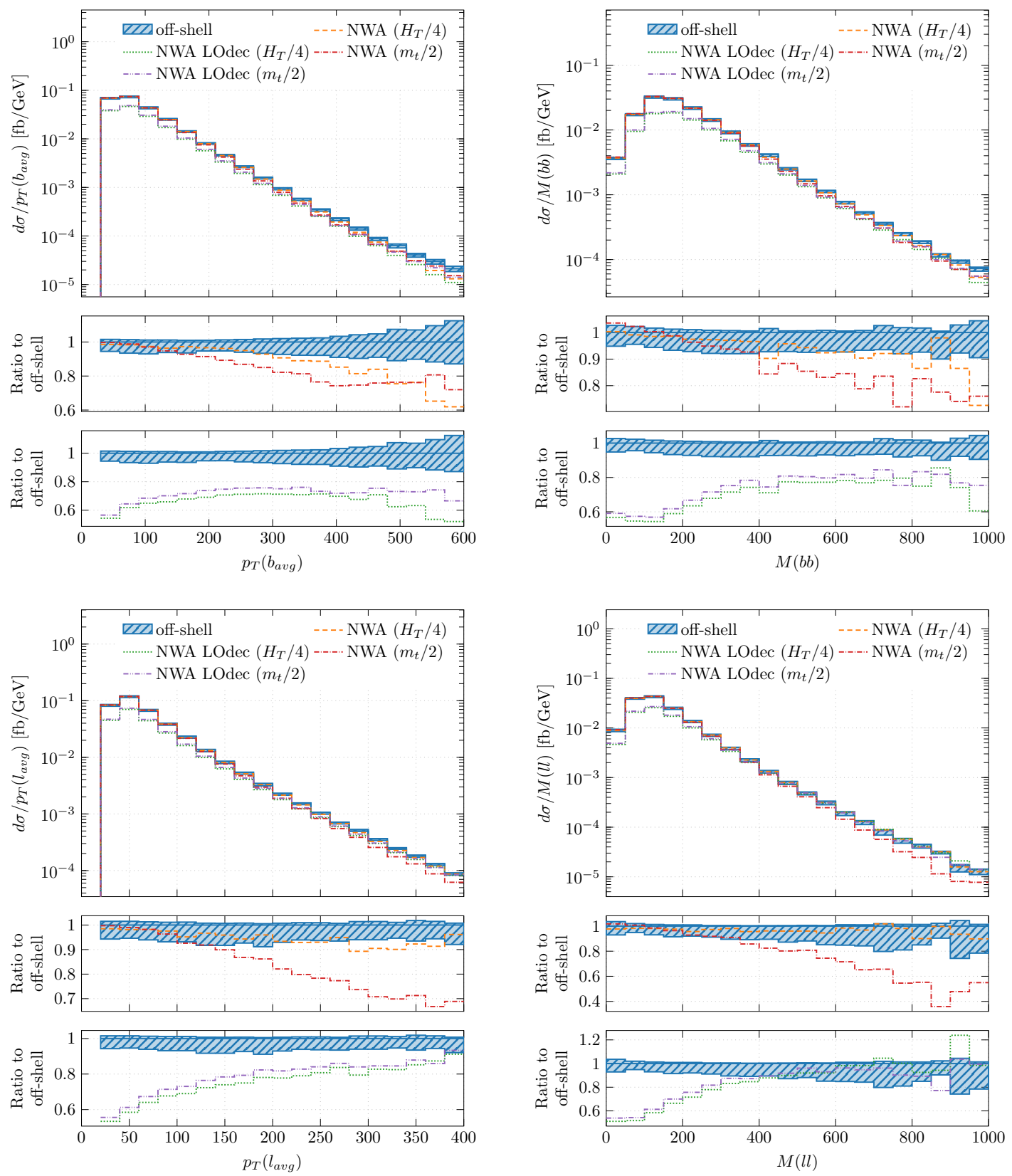

Figure 5. Differential cross section distribution as a function of the (averaged) transverse momentum of the $b$-jet, $p_{T}\left(b_{\text {avg }}\right)$, and charged lepton, $p_{T}\left(\ell_{\text {avg }}\right)$, as well as the invariant mass of the two $b$-jets, $M(b b)$, and two charged leptons system, $M(\ell \ell)$, for the $p p \rightarrow e^{+} \nu_{e} \mu^{-} \bar{\nu}_{\mu} b \bar{b} \gamma$ process at the LHC run II with $\sqrt{s}=13 \mathrm{TeV}$. The CT14 PDF set is employed. 

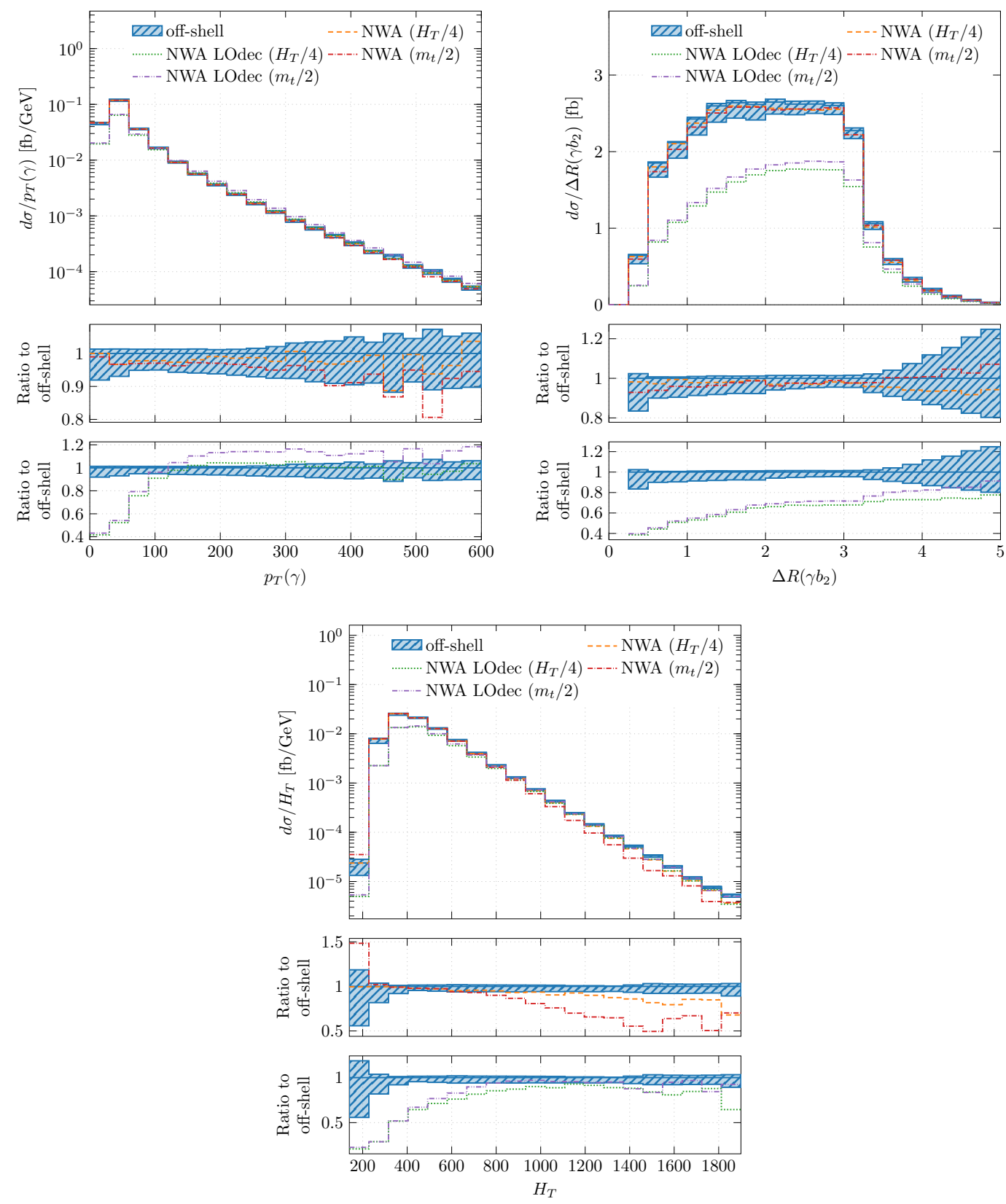

Figure 6. Differential cross section distribution as a function of the transverse momentum of the photon, $p_{T}(\gamma)$, the separation of the photon and the softest $b$-jet in the rapidity-azimuthal angle plane, $\Delta R\left(\gamma b_{2}\right)$, and the total transverse momentum of the $e^{+} \nu_{e} \mu^{-} \bar{\nu}_{\mu} b \bar{b} \gamma$ system, $H_{T}$ for the $p p \rightarrow e^{+} \nu_{e} \mu^{-} \bar{\nu}_{\mu} b \bar{b} \gamma$ process at the LHC run II with $\sqrt{s}=13 \mathrm{TeV}$. The CT14 PDF set is employed. 
Due to the matching ambiguity stemming from the presence of two $b$-jets the upper bound of $153 \mathrm{GeV}$ does not necessarily need to be obeyed. Choosing, however, the smallest $M\left(b e^{+}\right)$ for each event will guarantee that $M\left(b e^{+}\right)_{\min } \leq M\left(b e^{+}\right)$and that the kinematical endpoint of the distribution is preserved. For off-shell top quarks this kinematic limit is smeared, and furthermore, additional NLO QCD radiation and photon emission affect this region. Nevertheless there is a sharp fall of the cross section around $153 \mathrm{GeV}$. At NLO in QCD depending on the scale choice we can observe large and negative finite top quark width effects of the order of $40 \%-60 \%$, which are way above the theoretical uncertainties in that region. At the same time we can see that the $\mathrm{NWA}_{\text {LOdecay }}$ predictions are unable to correctly describe the observable in the whole plotted region. Not only the overall normalisation, but also the shape of the $M\left(b e^{+}\right)_{\text {min }}$ distribution can not be predicted by this approach. In the same manner the peak of the $M\left(t_{\mathrm{avg}}\right)$ distribution is smeared by the off-shell top quark effects and additional gluon and photon radiations. We note here, that the top and anti-top quarks are reconstructed from their decay products assuming the exact $W$ gauge boson reconstruction and perfect $b$ and $\bar{b}$ tagging efficiency. The full NWA results are consistently outside of the theoretical uncertainty band and finite top quark width effects are ranging from almost $+100 \%$ to $-100 \%$. For the NWA LOdecay case non-factorisable corrections are even larger reaching $150 \% . M\left(b e^{+}\right)_{\min }$ and $M\left(t_{\mathrm{avg}}\right)$ belong to the first class of observables potentially susceptible to the modelling of the top quark decays, i.e. the observables with kinematical threshold and edges.

In figure 5 we exhibit differential cross section distribution as a function of the (averaged) transverse momentum of the $b$-jet, $p_{T}\left(b_{\text {avg }}\right)$, and charged lepton, $p_{T}\left(\ell_{\text {avg }}\right)$. Also shown are the invariant mass of the two $b$-jets, $M(b \bar{b})$, and the invariant mass of the two charged leptons, $M(\ell \ell)$. For this class of observables the finite top quark width effects will appear in the high $p_{T}$ tails and for large values of the corresponding invariant mass differential distributions. Specifically, for the $b$-jet kinematics we have non-factorisable corrections of the order of $30 \%-40 \%$ independently of the scale choice. Similar effects are observed for the charged leptons for $\mu_{0}=m_{t} / 2$. On the other hand, for the dynamical scale choice they are negligible and within the theoretical uncertainties. Once again the $\mathrm{NWA}_{\text {LOdecay }}$ is not adequate to describe these observables. They fail already in the low $p_{T}$ regions.

Finally, in figure 6 we display differential cross section distribution as a function of the transverse momentum of the photon, $p_{T}(\gamma)$, the separation of the photon and the softest $b$-jet in the rapidity-azimuthal angle plane, $\Delta R\left(\gamma b_{2}\right)$, and the total transverse momentum of the $e^{+} \nu_{e} \mu^{-} \bar{\nu}_{\mu} b \bar{b} \gamma$ system, $H_{T}$. These three observables are well known for being sensitive to physics beyond the SM, see e.g. refs. $[7,11]$. For the $p_{T}(\gamma)$ distribution the finite top quark width effects are small and within theoretical uncertainties. Thus, for this particular differential cross section the full NWA description would be sufficient. Likewise for the angular distribution $\Delta R\left(\gamma b_{2}\right)$. Generally speaking, for all dimensionless observables, that we have studied, negligible non-factorisable corrections have been observed. Large top quark off-shell effects are estimated for the last observable $H_{T}$. They are in the range of $-50 \%$ to $+50 \%$ for $\mu_{0}=m_{t} / 2$ and up to $25 \%$ for $\mu_{0}=H_{T} / 4$. This of course is not surprising since $H_{T}$ comprises both $p_{T}\left(b_{1}\right), p_{T}\left(b_{2}\right)$ and $p_{T}\left(\ell_{1}\right), p_{T}\left(\ell_{2}\right)$ among others. On the other hand, the $\mathrm{NWA}_{\text {LOdecay }}$ can be disregard because it is consistently unable to describe correctly the shape of various observables. 
We can summarise this part by concluding that among all observables that we have examined only dimensionful observables are sensitive to non-factorizable top quark corrections that imply a cross-talk between production and decays of top quarks. We could identify two categories. The first class comprises observables with kinematical thresholds or edges. Such observables should be carefully examined in the vicinity of these thresholds/edges. The second class consists of dimensionful observables in the high $p_{T}$ regions. On the contrary, dimensionless observables like angular distributions do not seem to be very sensitive to the top quark off-shell effects.

\subsection{Double-, single- and non-resonant phase-space regions}

To understand better why some observables are more sensitive to the top quark off-shell effects than the other we investigate the contribution of double-, single- and non-resonant regions to the integrated and differential cross sections for the full off-shell case. This should qualitatively show us the importance of particular contributions and their overall distribution for particular phase space regions. To identify these contributions we have generalised the method introduced in ref. [33] and further discussed in e.g. [81, 82]. We first identify the following three different resonance histories

$$
\begin{aligned}
& \text { (i) } t=W^{+}\left(\rightarrow e^{+} \nu_{e}\right) b \quad \text { and } \quad \bar{t}=W^{-}\left(\rightarrow \mu^{-} \bar{\nu}_{\mu}\right) \bar{b} \text {, } \\
& \text { (ii) } t=W^{+}\left(\rightarrow e^{+} \nu_{e}\right) b \gamma \quad \text { and } \quad \bar{t}=W^{-}\left(\rightarrow \mu^{-} \bar{\nu}_{\mu}\right) \bar{b} \text {, } \\
& \text { (iii) } \quad t=W^{+}\left(\rightarrow e^{+} \nu_{e}\right) b \quad \text { and } \quad \bar{t}=W^{-}\left(\rightarrow \mu^{-} \bar{\nu}_{\mu}\right) \bar{b} \gamma \text {. }
\end{aligned}
$$

These three categories are not sufficient if NLO QCD calculations are considered. Therefore, in this case an additional resolved light jet (if present) has to be incorporated into the list. In practice, to closely mimic what is done on the experimental side only the light jet that passes all the cuts, that we have also applied for the $b$-jets, is added to the resonance history. In this case a total of 9 different possibilities have to be considered. We compute for each history the following quantity

$$
Q=\left|M(t)-m_{t}\right|+\left|M(\bar{t})-m_{t}\right|,
$$

where $M(t)$ and $M(\bar{t})$ are invariant masses of the top and anti-top quark respectively, where their momenta are reconstructed from the decay products assuming exact $W$ gauge boson reconstruction and perfect $b$ and $\bar{b}$-tagging efficiencies. Finally, we pick the history that minimises the $Q$ value. Once the history is determined, the check whether $t$ and $\bar{t}$ are off-shell or on-shell is performed. Specifically, we define the double-resonant (DR) region, when both $t$ and $\bar{t}$ are on-shell, via the following condition

$$
\left|M(t)-m_{t}\right|<n \Gamma_{t}, \quad \text { and } \quad\left|M(\bar{t})-m_{t}\right|<n \Gamma_{t} .
$$

There are two single-resonant (SR) regions that are given by

$$
\left|M(t)-m_{t}\right|<n \Gamma_{t}, \quad \text { and } \quad\left|M(\bar{t})-m_{t}\right|>n \Gamma_{t},
$$

or

$$
\left|M(t)-m_{t}\right|>n \Gamma_{t}, \quad \text { and } \quad\left|M(\bar{t})-m_{t}\right|<n \Gamma_{t} .
$$



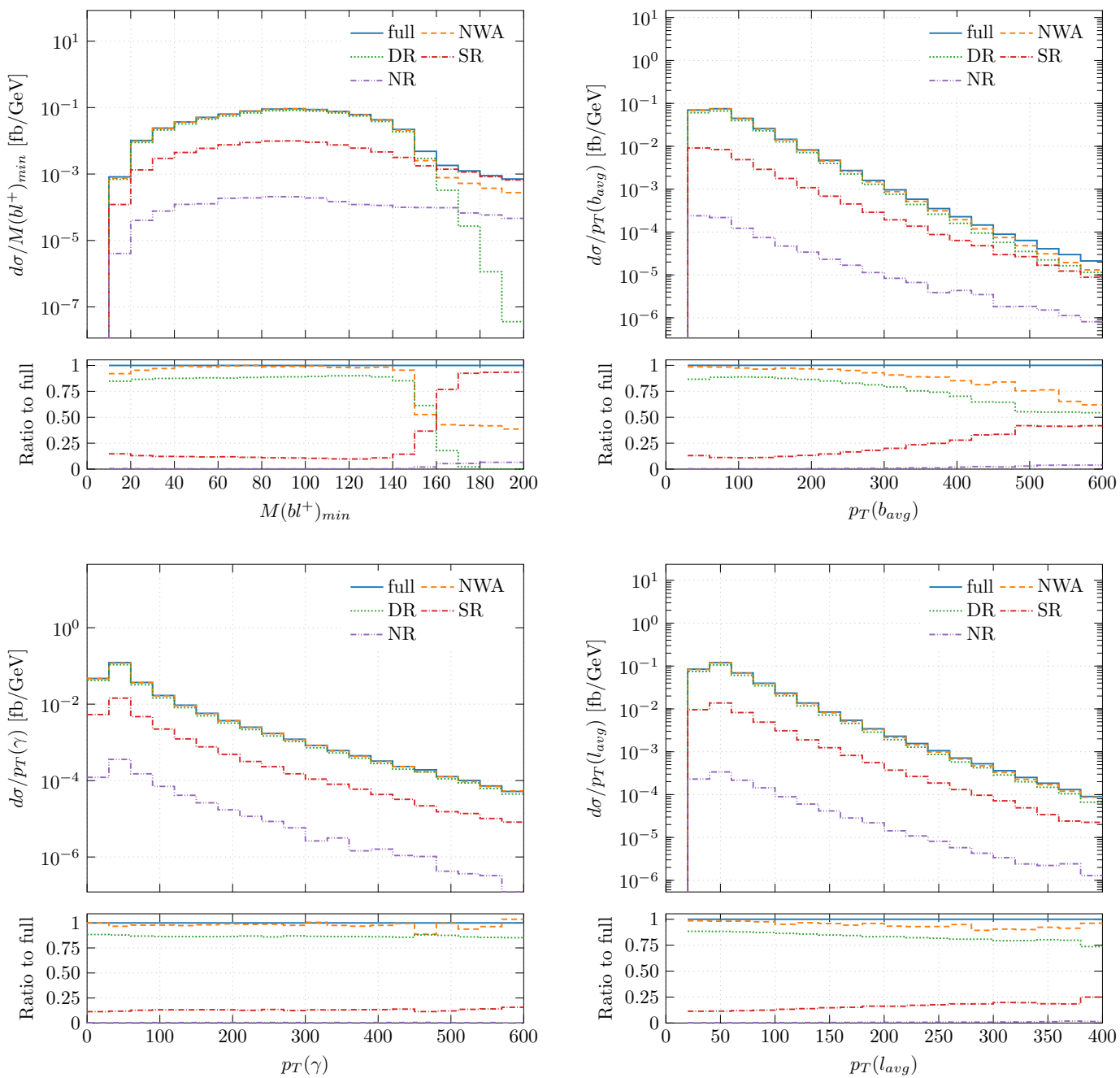

Figure 7. The $p p \rightarrow e^{+} \nu_{e} \mu^{-} \bar{\nu}_{\mu} b \bar{b} \gamma$ differential cross section distribution as a function of the minimum invariant mass of the $b$-jet and the positron, the (averaged) transverse momentum of the $b$-jet, the hard photon and the (averaged) charged lepton at the LHC run II with $\sqrt{s}=13 \mathrm{TeV}$. The upper plots show absolute NLO QCD predictions for DR, SR and NR regions. Also shown are NLO results for full off-shell and NWA case. The ratios of these contributions to the full off-shell result are also shown. Results are given for $\mu_{0}=H_{T} / 4$ and the CT14 PDF set.

Finally, the non-resonant (NR) region is chosen according to

$$
\left|M(t)-m_{t}\right|>n \Gamma_{t}, \quad \text { and } \quad\left|M(\bar{t})-m_{t}\right|>n \Gamma_{t} .
$$

The boundary parameter, which determines the size of the resonant region for each reconstructed top quark, has been set to $n=15$. This corresponds to the following condition for the DR region: $M(t) \in(152.9,193.5) \mathrm{GeV}$ and $M(\bar{t}) \in(152.9,193.5) \mathrm{GeV}$. The exact value of the boundary parameter is of course arbitrary. In the literature more stringent conditions, like for example $n=10, n=5$, have also been applied, see e.g. [33, 81, 82]. Having used the above outlined procedure the contributions at the integrated cross section 

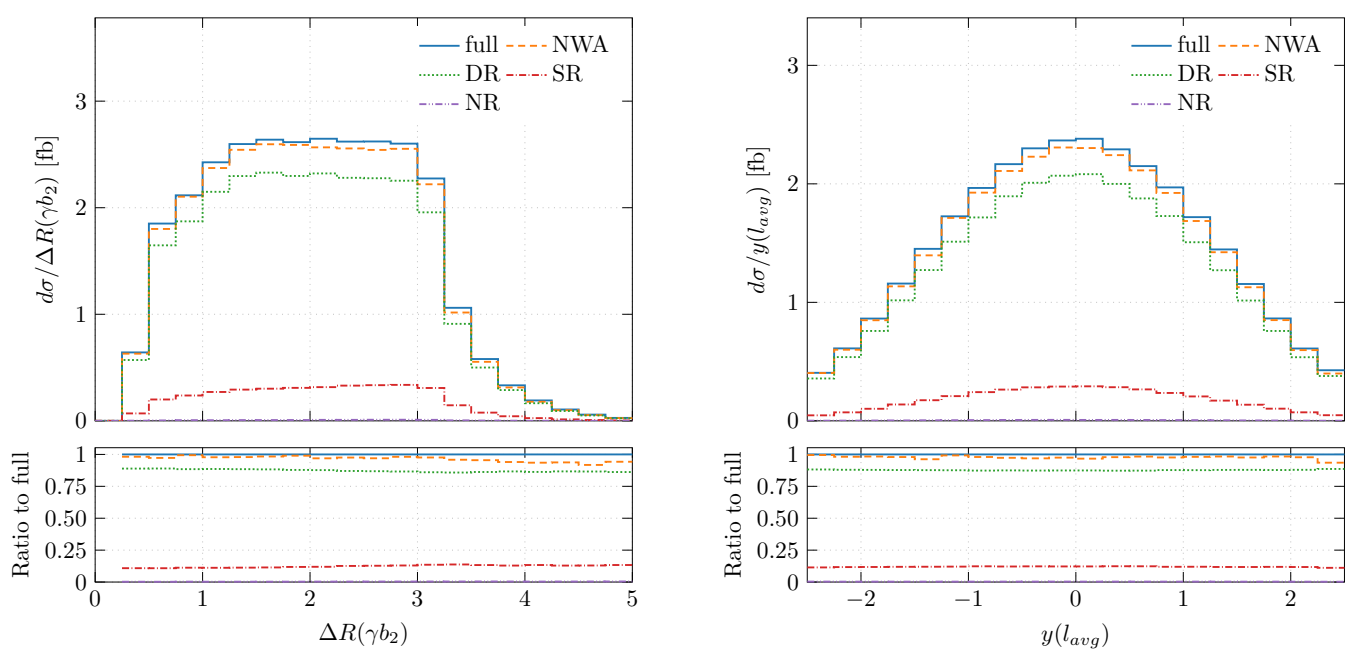

Figure 8. The $p p \rightarrow e^{+} \nu_{e} \mu^{-} \bar{\nu}_{\mu} b \bar{b} \gamma$ differential cross section distribution as a function of the rapidity-azimuthal angle separation between the hard photon and the softest $b$-jet and the (averaged) rapidity of the charged lepton at the LHC run II with $\sqrt{s}=13 \mathrm{TeV}$. The upper plots show absolute NLO QCD predictions for DR, SR and NR regions. Also shown are NLO results for full off-shell and NWA case. The ratios of these contributions to the full off-shell result are also shown. Results are given for $\mu_{0}=H_{T} / 4$ and the CT14 PDF set.

level for these three regions are given by

$$
\sigma_{\mathrm{DR}}^{\mathrm{NLO}}=6.57 \mathrm{fb}, \quad \sigma_{\mathrm{SR}}^{\mathrm{NLO}}=0.91 \mathrm{fb}, \quad \sigma_{\mathrm{NR}}^{\mathrm{NLO}}=0.02 \mathrm{fb} .
$$

The integrated fiducial cross section is dominated by the DR contributions. More than

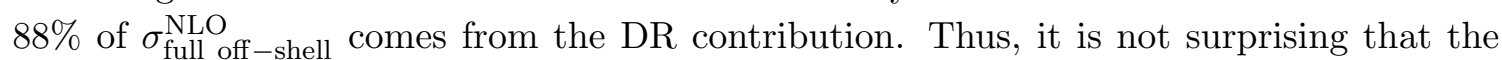
integrated cross section is not really sensitive to SR and NR contributions and therefore also to the top quark off-shell effects. The SR comprises only $12 \%$ of the full off-shell cross section at NLO in QCD whereas the contribution from the NR regions of the phase space is negligible, below $0.5 \% .{ }^{4}$ The situation, however, looks quite different at the differential level.

In figure 7 we show the differential cross section distribution as a function of $M\left(b e^{+}\right)_{\min }$, $M\left(t_{\text {avg }}\right), p_{T}\left(b_{\text {avg }}\right)$ and $p_{T}\left(\ell_{\text {avg }}\right)$. Also given are NLO results for the full off-shell and the full NWA cases. In the case of the $M\left(b e^{+}\right)_{\text {min }}$ distribution we observe that in the region that is not sensitive to the finite top quark width effects, i.e. $M\left(b e^{+}\right)_{\min }<153 \mathrm{GeV}$, the DR contribution is almost indistinguishable from the full off-shell and the full NWA result. However, once the vicinity of the kinematical cut-off is reached, these particular contributions begin to spread out. For $M\left(b e^{+}\right)_{\min } \geq 153 \mathrm{GeV}$ the SR part rapidly starts to dominate the full off-shell result and even the input from the NR regions of the phase space is larger than from the DR one. A similar effect can be observed for $p_{T}\left(b_{\text {avg }}\right)$. Even though in the high $p_{T}$ tail of the differential cross section distribution the SR part does not dominate the full off-shell result, its contribution increases to almost $50 \%$. On the other hand, the DR part contribution is greatly reduced, from around $85 \%$ down to about $50 \%$.

\footnotetext{
${ }^{4}$ Should we instead use $n=5$ we would get $\sigma_{\mathrm{DR}}^{\mathrm{NLO}}=4.82 \mathrm{fb}, \sigma_{\mathrm{SR}}^{\mathrm{NLO}}=2.50 \mathrm{fb}$ and $\sigma_{\mathrm{NR}}^{\mathrm{NLO}}=0.18 \mathrm{fb}$.
} 
For $p_{T}(\gamma)$ and $p_{T}\left(\ell_{\text {avg }}\right)$, which are evaluated with $\mu_{0}=H_{T} / 4$, in the whole plotted range the contribution from the SR part of the cross section is rather constant, of the order of $10 \%$ for $p_{T}(\gamma)$ and below $25 \%$ for $p_{T}\left(\ell_{\text {avg }}\right)$. Thus, it is consistently smaller than the DR one. This is obviously reflected in the small sensitivity of $d \sigma_{\text {full off }- \text { shell }}^{\mathrm{NLO}} / d X, X=p_{T}(\gamma), p_{T}\left(\ell_{\text {avg }}\right)$ to non-factorisable top quark corrections. We note at this point that all differential cross section distributions that are dimensionless in nature, which we have examined, received large $(90 \%)$ and constant contributions from the DR regions of the phase space in the whole kinematical range. At the same time the SR contribution was rather small (10\%), whereas the NR regions of the phase space were negligible. To illustrate these findings we present in figure 8 two examples, namely $\Delta R\left(\gamma b_{2}\right)$ and $y\left(\ell_{\text {avg }}\right)$. Consequently, dimensionless observables are rather insensitive to the finite top quark width effects.

We can conclude this part by stressing that observables that are sensitive to top quark off-shell effects have substantial contributions from the single top quark process. In these cases the best description is provided by the full off-shell calculation since it is free of ambiguities related to disentangling single and double resonant contributions.

\subsection{Photon radiation in the production and decays}

Finally, we would like to investigate the composition of photon emissions in the $t \bar{t} \gamma$ process, whether they come from production or decay stage. Having the full NWA implemented at the NLO in QCD level in the HELAC-NLO software such study can be straightforwardly performed. First, we discuss dimensionful observables like the transverse momentum of the hardest (in $p_{T}$ ) charged lepton, $p_{T}\left(\ell_{1}\right)$, and $b$-jet, $p_{T}\left(b_{1}\right)$, the total transverse momentum of the $e^{+} \nu_{e} \mu^{-} \bar{\nu}_{\mu} b \bar{b} \gamma$ system, $H_{T}$, and the transverse momentum of the hard photon, $p_{T}(\gamma)$. They are all exhibited in figure 9. We notice that in all four cases for the low values of the transverse momentum the differential distributions are dominated by photon emission in the decay stage. Specifically, in these regions more than $50 \%$ of photons come from top quark decays. For the $H_{T}$ observable this contribution reaches even $75 \%$. However, once the high $p_{T}$ region of these observables are probed, photon emission from the production part of the $t \bar{t} \gamma$ process dominates completely the full results. Thus, for all four observables setting high $p_{T}$ cut would eliminate or at least substantially diminish the contribution from the hard photon in top quark decays.

In figure 10 we show differential cross section distribution as a function of $M\left(b \ell^{+}\right)_{\min }$ and $M\left(t_{\text {avg }}\right)$. Even though these two also belong to dimensionful observables photon radiation is distributed almost evenly between the production and decay part of the $t \bar{t} \gamma$ process. In the case of $M\left(b \ell^{+}\right)_{\min }$ the contribution from the $\mathrm{NWA}_{\gamma \text { decay }}$ part is at least $25 \%$ and can go up to about $60 \%$. Instead, for $M\left(t_{\mathrm{avg}}\right)$, rather constant $65 \%$ and $35 \%$ contributions can be seen respectively for the $\mathrm{NWA}_{\gamma \text { prod }}$ and $\mathrm{NWA}_{\gamma \text { decay }}$ case. Therefore, for both observable there is no straightforward way to decrease the contribution from the latter.

At last, in figure 11 dimensionless observables are displayed. In particular, we provide differential cross section distribution as a function of the rapidity-azimuthal angle separation between: the photon and the softer $b$-jet, $\Delta R\left(\gamma b_{2}\right)$, the photon and the hardest charged lepton, $\Delta R\left(\gamma \ell_{1}\right)$, and the photon and the softest charged lepton, $\Delta R\left(\gamma \ell_{2}\right)$. Also shown is the rapidity of the hardest charged lepton, $y\left(\ell_{1}\right)$. For all three rapidity-azimuthal 

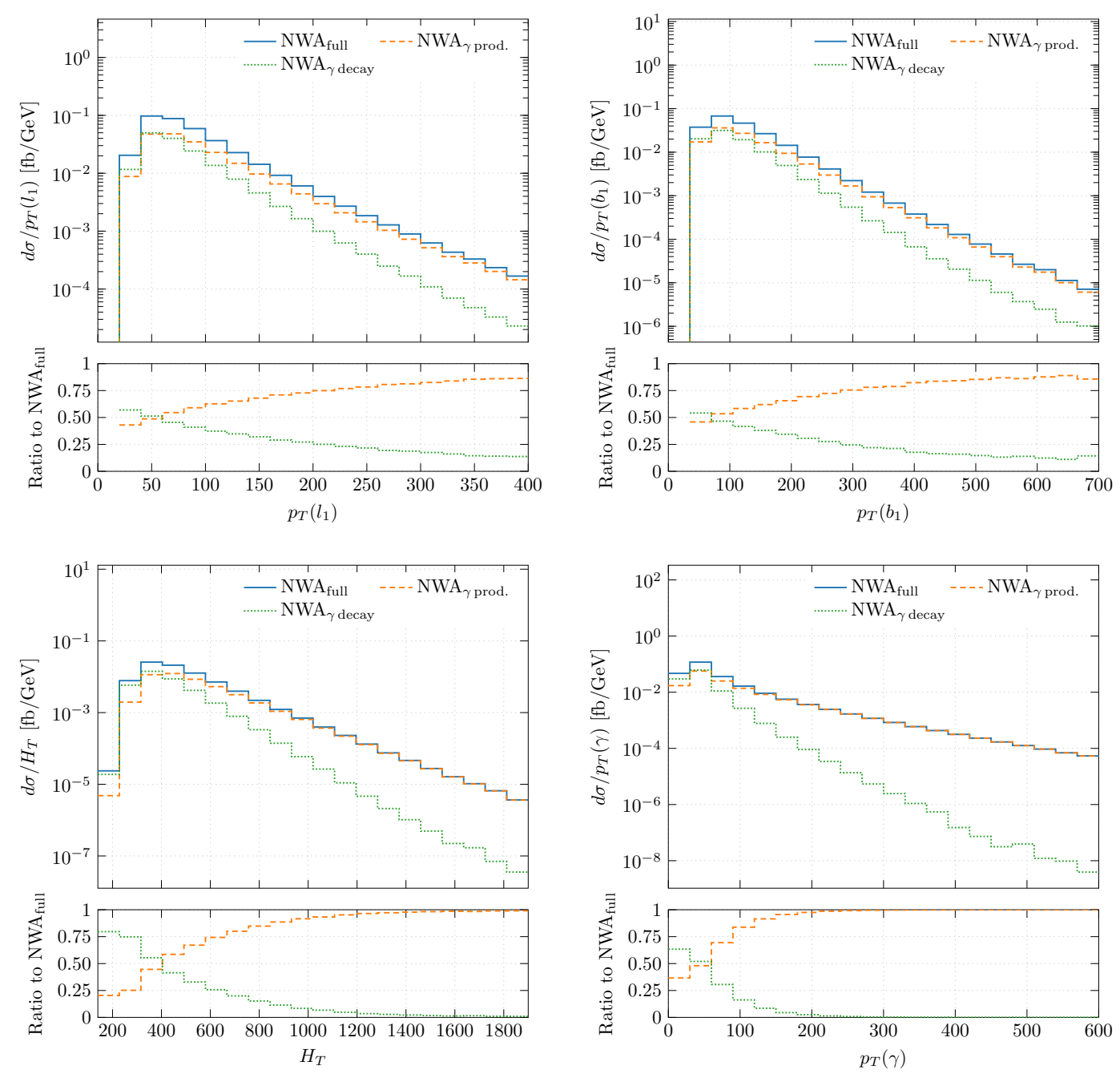

Figure 9. The $p p \rightarrow e^{+} \nu_{e} \mu^{-} \bar{\nu}_{\mu} b \bar{b} \gamma$ differential cross section distribution as a function of the transverse momentum of the hardest charged lepton, $p_{T}\left(\ell_{1}\right)$, and $b$-jet, $p_{T}\left(b_{1}\right)$, the total transverse momentum of the $e^{+} \nu_{e} \mu^{-} \bar{\nu}_{\mu} b \bar{b} \gamma$ system, $H_{T}$, and the transverse momentum of the isolated photon, $p_{T}(\gamma)$, at the LHC run II with $\sqrt{s}=13 \mathrm{TeV}$. The upper plots show absolute NLO QCD predictions in the full NWA together with fraction of events originating from photon radiation in the production,

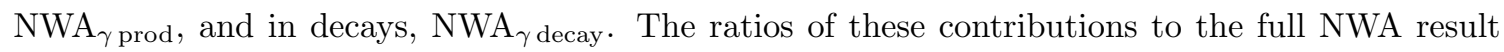
are also shown. Results are given for $\mu_{0}=H_{T} / 4$ and the CT14 PDF sets are employed.

angle separations up to about $\Delta R \lesssim 1$ photon radiation in top quark decays dominates the corresponding differential cross section in that region of the phase-space. For $\Delta R\left(\gamma \ell_{1}\right)$ and $\Delta R\left(\gamma \ell_{2}\right)$ for example more than $80 \%$ of all emitted photons originate in $t \rightarrow b e^{+} \nu_{e} \gamma$ and $\bar{t} \rightarrow \bar{b} \mu^{-} \bar{\nu}_{\mu} \gamma$ processes. For $\Delta R \gtrsim 1$ the $\mathrm{NWA}_{\gamma \text { prod }}$ contribution surpasses the $\mathrm{NWA}_{\gamma}$ decay one, although the latter stays at the substantial level. Specifically, we can observe contributions of the order of $20 \%-40 \%$. Finally, as far as $y\left(\ell_{1}\right)$ is concerned rather constant contributions from both parts are visible for $y\left(\ell_{1}\right) \in\langle-2.5,2.5\rangle$, i.e. about $40 \%$ for the $\mathrm{NWA}_{\gamma \text { decay }}$ case and around $60 \%$ for $\mathrm{NWA}_{\gamma \text { prod }}$. 

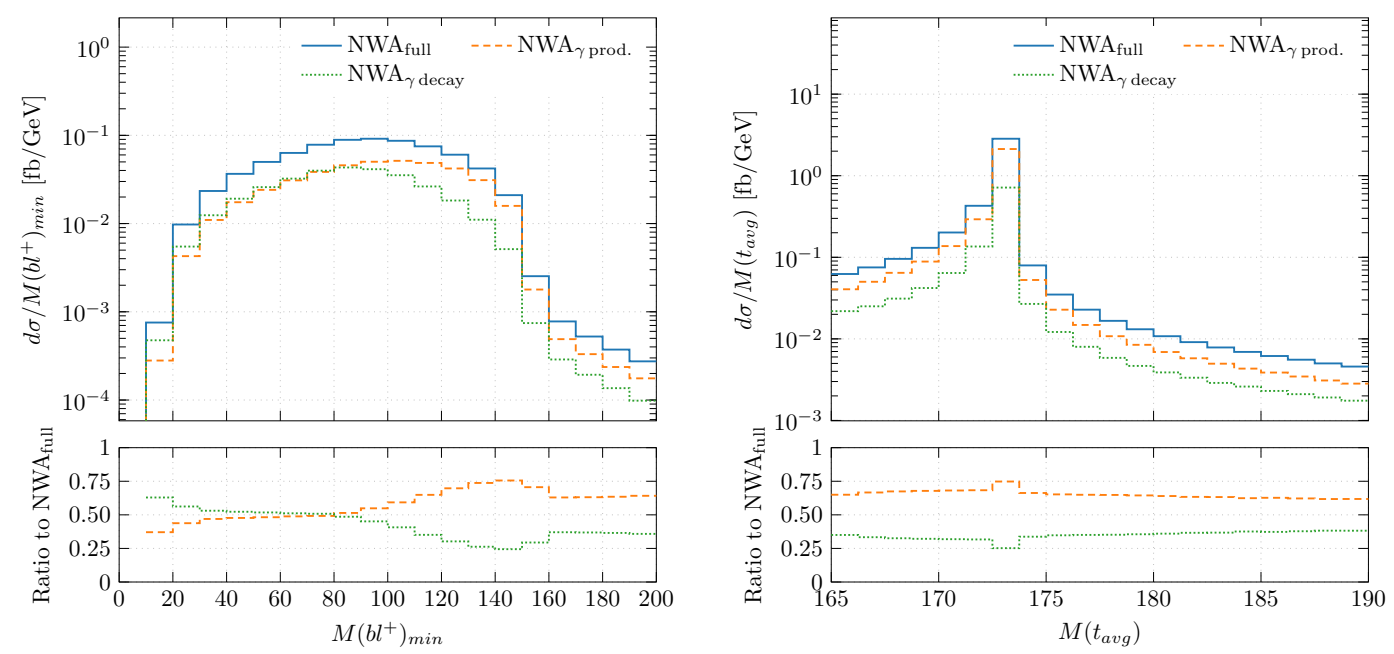

Figure 10. The $p p \rightarrow e^{+} \nu_{e} \mu^{-} \bar{\nu}_{\mu} b \bar{b} \gamma$ differential cross section distribution as a function of the minimum invariant mass of the positron and $b$-jet, $M\left(b \ell^{+}\right)_{\min }$, and the (averaged) invariant mass of the reconstructed top quark, $M\left(t_{\text {avg }}\right)$, at the LHC run II with $\sqrt{s}=13 \mathrm{TeV}$. The upper plots show absolute NLO QCD predictions in the full NWA together with fraction of events originating from photon radiation in the production, $\mathrm{NWA}_{\gamma \text { prod }}$, and in decays, $\mathrm{NWA}_{\gamma \text { decay }}$. The ratios of these contributions to the full NWA result are also shown. Results are given for $\mu_{0}=H_{T} / 4$ and the CT14 PDF sets are employed.

\section{Conclusions}

In this paper we have presented a comparative study of various approaches for modelling of the $e^{+} \nu_{e} \mu^{-} \bar{\nu}_{\mu} b \bar{b} \gamma$ final state in $t \bar{t} \gamma$ production at the LHC. We compared the fully realistic description as given by a complete calculation with the one provided by the NWA. In the latter case two versions have been examined: the full NWA and the $\mathrm{NWA}_{\text {LOdecay }}$ (i.e. NWA with LO decays of top quarks and photon radiation in the production stage only). When comparing full off-shell and full NWA results we confirmed that for the integrated cross sections the finite top quark width effects are small, of the order of $\mathcal{O}\left(\Gamma_{t} / m_{t}\right)$. We have shown, however, that they are strongly enhanced for more exclusive (dimensionful) observables even up to $60 \%$. On the contrary, dimensionless observables like angular differential cross section distributions appear to be relatively insensitive to the top quark off-shell effects. Furthermore, we have revealed that the NWA $_{\text {LOdecay }}$ approach is simply not adequate in describing the $e^{+} \nu_{e} \mu^{-} \bar{\nu}_{\mu} b \bar{b} \gamma$ process neither at the integrated level nor at the differential one. Not only the NLO QCD corrections to top quark decays have to be incorporated but also hard photon emission from the top quark decays must be included.

To better understand the sensitivity of kinematic observables to the non-factorisable top quark corrections, we have devised the procedure to divide the full fiducial phase space of the $e^{+} \nu_{e} \mu^{-} \bar{\nu}_{\mu} b \bar{b} \gamma$ process into double-, single- and non-resonant parts. We concluded that observables that are sensitive to top quark off-shell effects have substantial contribution from the single top quark process. In these cases the best description is provided by the full off-shell calculation since it is free of ambiguities related to disentangling single and double resonant contributions. 

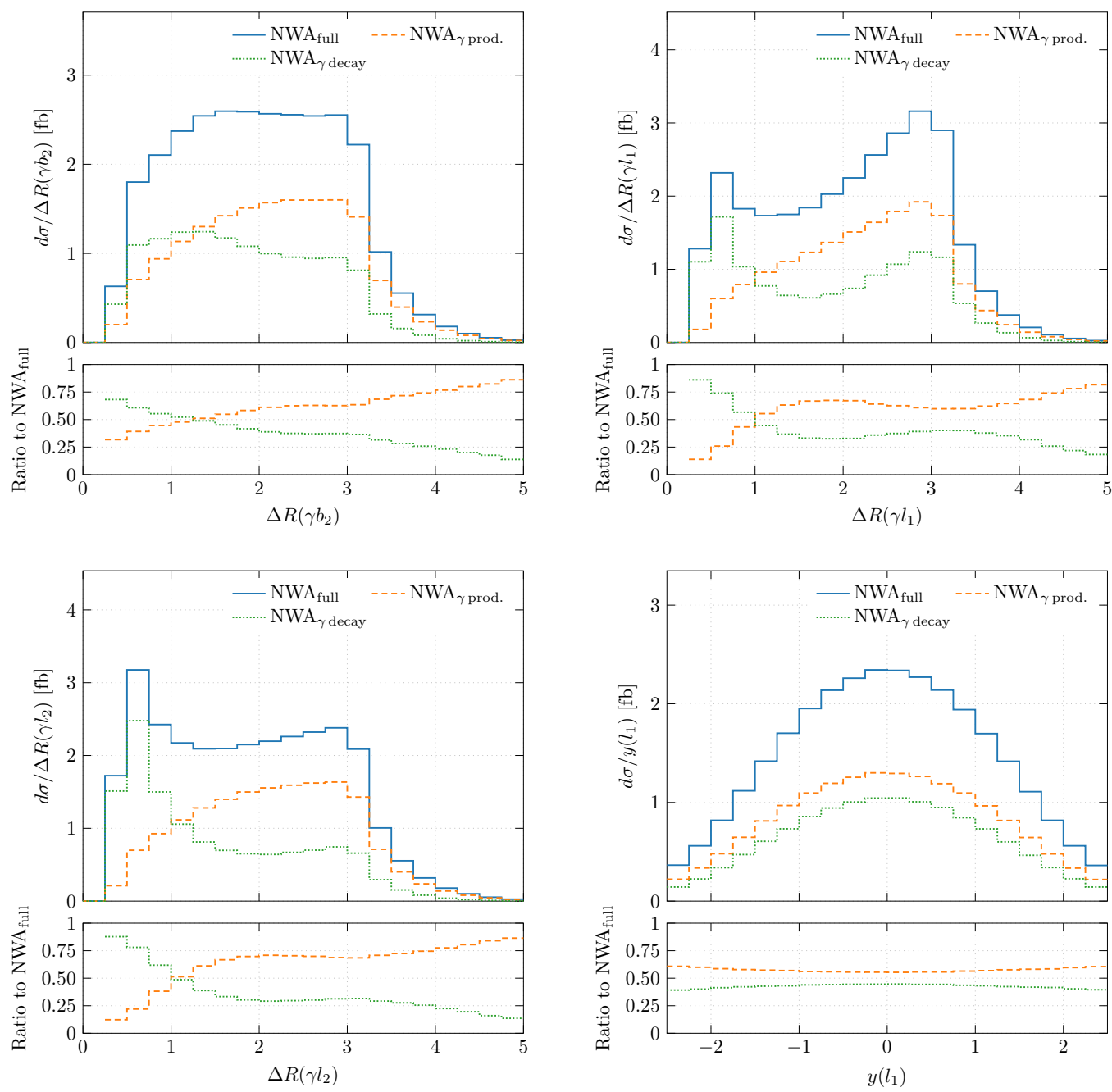

Figure 11. The $p p \rightarrow e^{+} \nu_{e} \mu^{-} \bar{\nu}_{\mu} b \bar{b} \gamma$ differential cross section distribution as a function of the rapidity-azimuthal angle separation between: the photon and the softer b-jet, $\Delta R\left(\gamma b_{2}\right)$, the photon and the hardest charged lepton, $\Delta R\left(\gamma \ell_{1}\right)$, as well as the photon and the softest charged lepton, $\Delta R\left(\gamma \ell_{2}\right)$ at the LHC run II with $\sqrt{s}=13 \mathrm{TeV}$. Also shown is the differential cross section distribution as a function of the rapidity of the hardest charged lepton, $y\left(\ell_{1}\right)$, The upper plots show absolute NLO QCD predictions in the full NWA together with fraction of events originating from photon radiation in the production, $\mathrm{NWA}_{\gamma \text { prod }}$, and in decays, $\mathrm{NWA}_{\gamma \text { decay }}$. The ratios of these contributions to the full NWA result are also shown. Results are given for $\mu_{0}=H_{T} / 4$ and the CT14 PDF sets are employed. 
In addition, we investigate fractions of events where the photon is radiated either in the production or in the decay stage. We find that large fraction of isolated photons comes from the decays of top quarks. Based on our findings, selection criteria might be developed to reduce such contributions that constitute a background for the measurement of the anomalous couplings in the $t \bar{t} \gamma$ vertex. For example, for the transverse momentum of the hardest lepton, $b$-jet and the hard photon as well as the total transverse momentum of the $e^{+} \nu_{e} \mu^{-} \bar{\nu}_{\mu} b \bar{b} \gamma$ system such kinematical cuts could be introduced. However, many other observables have rather constant and substantial contribution from the hard photon in the top quark decays. Thus, a simple procedure to decrease such contributions would not be possible for them. Here, the most important examples are the minimum invariant mass of the $b$-jet and the positron as well as the (averaged) invariant mass of the top quark.

Last but not least, on the technical side we have implemented the full NWA approach into the HelaC-Nlo Monte Carlo program. This has helped us to provide theoretical predictions for the full NWA and the NWALOdecay cases for the $t \bar{t} \gamma$ production at the LHC. Such automation opens a new path for performing higher order calculations for more complex processes at the LHC such as $t \bar{t} b \bar{b}, t \bar{t} j j$ and $t \bar{t} t \bar{t}$ where the top-quark decays are realistically simulated through the NWA approach.

\section{Acknowledgments}

We thank Rene Poncelet for a cross-check with the results of ref. [39].

The research of G.B. was supported by grant K 125105 of the National Research, Development and Innovation Office in Hungary. G.B. also thanks the Institute for Theoretical Particle Physics and Cosmology of RWTH Aachen University for financial support and hospitality during the completion of this work.

The work of H.B.H. has received funding from the European Research Council (ERC) under the European Union's Horizon 2020 research and innovation programme (grant agreement No 772099).

The work of M.W. and T.W. was supported in part by the German Research Foundation (DFG) Individual Research Grant: Top-Quarks under the LHCs Magnifying Glass: From Process Modelling to Parameter Extraction and in part by the DFG Collaborative Research Centre/Transregio project CRC/TRR 257: P3H - Particle Physics Phenomenology after the Higgs Discovery.

Support by a grant of the Bundesministerium für Bildung und Forschung (BMBF) is additionally acknowledged.

Simulations were performed with computing resources granted by RWTH Aachen University under project rwth0414.

Open Access. This article is distributed under the terms of the Creative Commons Attribution License (CC-BY 4.0), which permits any use, distribution and reproduction in any medium, provided the original author(s) and source are credited. 


\section{References}

[1] CDF collaboration, Evidence for $t \bar{t} \gamma$ production and measurement of $\sigma_{t} \bar{t} \gamma / \sigma_{t} \bar{t}$, Phys. Rev. D 84 (2011) 031104 [arXiv: 1106.3970] [INSPIRE].

[2] ATLAS collaboration, Observation of top-quark pair production in association with a photon and measurement of the $t \bar{t} \gamma$ production cross section in pp collisions at $\sqrt{s}=7$ TeV using the ATLAS detector, Phys. Rev. D 91 (2015) 072007 [arXiv:1502.00586] [InSPIRE].

[3] ATLAS collaboration, Measurement of the $t \bar{t} \gamma$ production cross section in proton-proton collisions at $\sqrt{s}=8$ TeV with the ATLAS detector, JHEP 11 (2017) 086 [arXiv: 1706.03046] [INSPIRE].

[4] CMS collaboration, Measurement of the semileptonic $t \bar{t}+\gamma$ production cross section in $p p$ collisions at $\sqrt{s}=8 \mathrm{Te}$, JHEP 10 (2017) 006 [arXiv:1706.08128] [INSPIRE].

[5] ATLAS collaboration, Measurements of inclusive and differential fiducial cross-sections of $t \bar{t} \gamma$ production in leptonic final states at $\sqrt{s}=13$ TeV in ATLAS, Eur. Phys. J. C 79 (2019) 382 [arXiv: 1812.01697] [INSPIRE].

[6] ATLAS collaboration, Measurements of inclusive and differential cross-sections of $t \bar{t} \gamma$ production in the e $\mu$ channel at 13 TeV with the ATLAS detector, ATLAS-CONF-2019-042 (2019).

[7] U. Baur, M. Buice and L.H. Orr, Direct measurement of the top quark charge at hadron colliders, Phys. Rev. D 64 (2001) 094019 [hep-ph/0106341] [INSPIRE].

[8] CDF collaboration, Exclusion of exotic top-like quarks with $-4 / 3$ electric charge using jet-charge tagging in single-lepton t $\bar{t}$ events at CDF, Phys. Rev. D 88 (2013) 032003 [arXiv: 1304.4141] [INSPIRE].

[9] ATLAS collaboration, Measurement of the top quark charge in pp collisions at $\sqrt{s}=7$ TeV with the ATLAS detector, JHEP 11 (2013) 031 [arXiv:1307.4568] [INSPIRE].

[10] J.A. Aguilar-Saavedra, A minimal set of top anomalous couplings, Nucl. Phys. B 812 (2009) 181 [arXiv:0811.3842] [INSPIRE].

[11] U. Baur, A. Juste, L.H. Orr and D. Rainwater, Probing electroweak top quark couplings at hadron colliders, Phys. Rev. D 71 (2005) 054013 [hep-ph/0412021] [INSPIRE].

[12] A.O. Bouzas and F. Larios, Electromagnetic dipole moments of the Top quark, Phys. Rev. D 87 (2013) 074015 [arXiv: 1212.6575] [INSPIRE].

[13] M. Schulze and Y. Soreq, Pinning down electroweak dipole operators of the top quark, Eur. Phys. J. C 76 (2016) 466 [arXiv:1603.08911] [InSPIRE].

[14] O. Bessidskaia Bylund et al., Probing top quark neutral couplings in the standard model effective field theory at NLO in QCD, JHEP 05 (2016) 052 [arXiv:1601.08193] [INSPIRE].

[15] G. Bevilacqua et al., Precise predictions for $t \bar{t} \gamma / t \bar{t}$ cross section ratios at the LHC, JHEP 01 (2019) 188 [arXiv: 1809.08562] [INSPIRE].

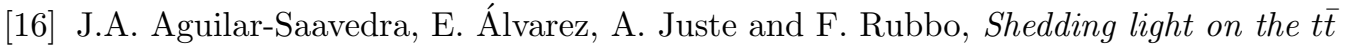
asymmetry: the photon handle, JHEP 04 (2014) 188 [arXiv:1402.3598] [INSPIRE].

[17] J.A. Aguilar-Saavedra, Single lepton charge asymmetries in $t \bar{t}$ and $t \bar{t} \gamma$ production at the LHC, Eur. Phys. J. C 78 (2018) 434 [arXiv:1802.05721] [InSPIRE].

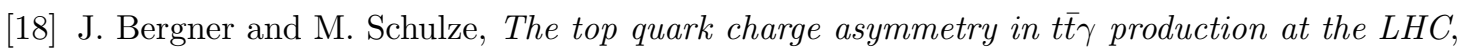
Eur. Phys. J. C 79 (2019) 189 [arXiv:1812.10535] [INSPIRE]. 
[19] P.-F. Duan et al., QCD corrections to associated production of $t \bar{t} \gamma$ at hadron colliders, Phys. Rev. D 80 (2009) 014022 [arXiv:0907.1324] [INSPIRE].

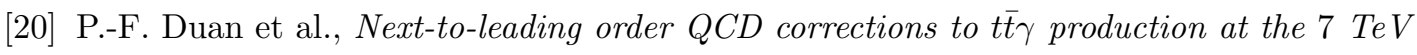
LHC, Chin. Phys. Lett. 28 (2011) 111401 [arXiv:1110.2315] [InSPIRE].

[21] F. Maltoni, D. Pagani and I. Tsinikos, Associated production of a top-quark pair with vector

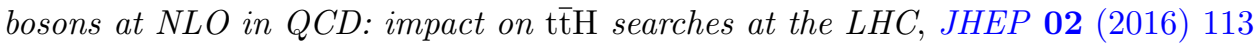
[arXiv: 1507.05640] [INSPIRE].

[22] P.-F. Duan et al., Electroweak corrections to top quark pair production in association with a hard photon at hadron colliders, Phys. Lett. B 766 (2017) 102 [arXiv:1612.00248] [INSPIRE].

[23] A. Kardos and Z. Trócsányi, Hadroproduction of t $t \bar{t}$ pair in association with an isolated photon at NLO accuracy matched with parton shower, JHEP 05 (2015) 090 [arXiv: 1406.2324] [INSPIRE].

[24] S. Frixione, P. Nason and C. Oleari, Matching NLO QCD computations with Parton Shower simulations: the POWHEG method, JHEP 11 (2007) 070 [arXiv:0709.2092] [INSPIRE].

[25] S. Alioli, P. Nason, C. Oleari and E. Re, A general framework for implementing NLO calculations in shower Monte Carlo programs: the POWHEG BOX, JHEP 06 (2010) 043 [arXiv: 1002.2581] [INSPIRE].

[26] G. Bevilacqua et al., HELAC-NLO, Comput. Phys. Commun. 184 (2013) 986 [arXiv:1110.1499] [INSPIRE].

[27] T. Sjöstrand et al., An introduction to PYTHIA 8.2, Comput. Phys. Commun. 191 (2015) 159 [arXiv: 1410.3012] [INSPIRE].

[28] M. Bahr et al., HERWIG++ physics and manual, Eur. Phys. J. C 58 (2008) 639 [arXiv: 0803.0883] [INSPIRE].

[29] K. Melnikov, M. Schulze and A. Scharf, QCD corrections to top quark pair production in association with a photon at hadron colliders, Phys. Rev. D 83 (2011) 074013 [arXiv: 1102.1967] [INSPIRE].

[30] G. Bevilacqua et al., Hard photons in hadroproduction of top quarks with realistic final states, JHEP 10 (2018) 158 [arXiv:1803.09916] [INSPIRE].

[31] A. van Hameren, C.G. Papadopoulos and R. Pittau, Automated one-loop calculations: a proof of concept, JHEP 09 (2009) 106 [arXiv:0903.4665] [INSPIRE].

[32] M. Czakon, C.G. Papadopoulos and M. Worek, Polarizing the dipoles, JHEP 08 (2009) 085 [arXiv: 0905.0883] [INSPIRE].

[33] N. Kauer and D. Zeppenfeld, Finite width effects in top quark production at hadron colliders, Phys. Rev. D 65 (2002) 014021 [hep-ph/0107181] [INSPIRE].

[34] C.F. Uhlemann and N. Kauer, Narrow-width approximation accuracy, Nucl. Phys. B 814 (2009) 195 [arXiv:0807.4112] [INSPIRE].

[35] W. Bernreuther, A. Brandenburg, Z.G. Si and P. Uwer, Top quark pair production and decay at hadron colliders, Nucl. Phys. B 690 (2004) 81 [hep-ph/0403035] [INSPIRE].

[36] K. Melnikov and M. Schulze, NLO QCD corrections to top quark pair production and decay at hadron colliders, JHEP 08 (2009) 049 [arXiv: 0907.3090] [INSPIRE].

[37] K. Melnikov, A. Scharf and M. Schulze, Top quark pair production in association with a jet: QCD corrections and jet radiation in top quark decays, Phys. Rev. D 85 (2012) 054002 [arXiv: 1111.4991] [INSPIRE]. 
[38] J.M. Campbell and R.K. Ellis, Top-quark processes at NLO in production and decay, J. Phys. G 42 (2015) 015005 [arXiv: 1204.1513] [INSPIRE].

[39] A. Behring et al., Higher order corrections to spin correlations in top quark pair production at the LHC, Phys. Rev. Lett. 123 (2019) 082001 [arXiv:1901.05407] [INSPIRE].

[40] V.S. Fadin, V.A. Khoze and A.D. Martin, How suppressed are the radiative interference effects in heavy instable particle production?, Phys. Lett. B 320 (1994) 141 [hep-ph/9309234] [INSPIRE].

[41] SM and NLO MULTILEG Working Group, SM MC Working Group collaboration, The SM and NLO multileg and SM MC working groups: summary report, arXiv:1203.6803 [INSPIRE].

[42] A. Denner, S. Dittmaier, S. Kallweit and S. Pozzorini, NLO QCD corrections to off-shell ttbar production at hadron colliders, PoS LL2012 (2012) 015 [arXiv: 1208.4053] [INSPIRE].

[43] R. Röntsch and M. Schulze, Constraining couplings of top quarks to the $Z$ boson in $t \bar{t}+Z$ production at the LHC, JHEP 07 (2014) 091 [Erratum ibid. 09 (2015) 132] [arXiv: 1404.1005] [INSPIRE].

[44] K. Melnikov and M. Schulze, NLO QCD corrections to top quark pair production in association with one hard jet at hadron colliders, Nucl. Phys. B 840 (2010) 129 [arXiv: 1004.3284] [INSPIRE].

[45] J.M. Campbell and R.K. Ellis, $t \bar{t} W^{ \pm}$production and decay at NLO, JHEP 07 (2012) 052 [arXiv: 1204.5678] [INSPIRE].

[46] A. Bredenstein, A. Denner, S. Dittmaier and S. Pozzorini, NLO QCD corrections to $p p \rightarrow t \bar{t} b \bar{b}+X$ at the LHC, Phys. Rev. Lett. 103 (2009) 012002 [arXiv:0905.0110] [INSPIRE].

[47] G. Bevilacqua et al., Assault on the NLO Wishlist: $p p \rightarrow t \bar{t} b \bar{b}$, JHEP 09 (2009) 109 [arXiv: 0907.4723] [INSPIRE].

[48] A. Bredenstein, A. Denner, S. Dittmaier and S. Pozzorini, NLO QCD corrections to top anti-top bottom anti-bottom production at the LHC: 2. Full hadronic results, JHEP 03 (2010) 021 [arXiv: 1001.4006] [INSPIRE].

[49] G. Bevilacqua, M. Czakon, C.G. Papadopoulos and M. Worek, Dominant QCD backgrounds in Higgs boson analyses at the LHC: a study of $p p \rightarrow t \bar{t}+2$ jets next-to-leading order, Phys. Rev. Lett. 104 (2010) 162002 [arXiv:1002.4009] [INSPIRE].

[50] G. Bevilacqua, M. Czakon, C.G. Papadopoulos and M. Worek, Hadronic top-quark pair production in association with two jets at next-to-leading order QCD, Phys. Rev. D 84 (2011) 114017 [arXiv:1108.2851] [INSPIRE].

[51] G. Bevilacqua and M. Worek, Constraining BSM physics at the LHC: four top final states with NLO accuracy in perturbative QCD, JHEP 07 (2012) 111 [arXiv: 1206.3064] [INSPIRE].

[52] R. Frederix, D. Pagani and M. Zaro, Large NLO corrections in $t \bar{t} W^{ \pm}$and $t \bar{t} t \bar{t}$ hadroproduction from supposedly subleading EW contributions, JHEP 02 (2018) 031 [arXiv: 1711.02116] [INSPIRE].

[53] M.V. Garzelli, A. Kardos and Z. Trócsányi, Hadroproduction of $t \bar{t} b \bar{b}$ final states at LHC: predictions at NLO accuracy matched with Parton Shower, JHEP 03 (2015) 083 [arXiv: 1408.0266] [INSPIRE].

[54] G. Bevilacqua, M.V. Garzelli and A. Kardos, $t \bar{t} b \bar{b}$ hadroproduction with massive bottom quarks with PowHel, arXiv:1709.06915 [INSPIRE]. 
[55] T. Ježo, J.M. Lindert, N. Moretti and S. Pozzorini, New NLOPS predictions for $t \bar{t}+b$-jet production at the LHC, Eur. Phys. J. C 78 (2018) 502 [arXiv: 1802.00426] [INSPIRE].

[56] A. Kanaki and C.G. Papadopoulos, HELAC: a package to compute electroweak helicity amplitudes, Comput. Phys. Commun. 132 (2000) 306 [hep-ph/0002082] [INSPIRE].

[57] A. Cafarella, C.G. Papadopoulos and M. Worek, Helac-phegas: a generator for all parton level processes, Comput. Phys. Commun. 180 (2009) 1941 [arXiv:0710.2427] [INSPIRE].

[58] G. Bevilacqua et al., Complete off-shell effects in top quark pair hadroproduction with leptonic decay at next-to-leading order, JHEP 02 (2011) 083 [arXiv:1012.4230] [INSPIRE].

[59] G. Bevilacqua, H.B. Hartanto, M. Kraus and M. Worek, Top quark pair production in association with a jet with next-to-leading-order QCD off-shell effects at the Large Hadron Collider, Phys. Rev. Lett. 116 (2016) 052003 [arXiv: 1509.09242] [INSPIRE].

[60] G. Bevilacqua, H.B. Hartanto, M. Kraus and M. Worek, Off-shell top quarks with one jet at the LHC: a comprehensive analysis at NLO QCD, JHEP 11 (2016) 098 [arXiv:1609.01659] [INSPIRE].

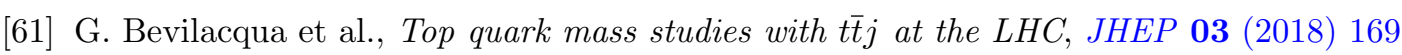
[arXiv: 1710.07515] [INSPIRE].

[62] G. Bevilacqua et al., Towards constraining dark matter at the LHC: higher order QCD predictions for $t \bar{t}+Z\left(Z \rightarrow \nu_{\ell} \bar{\nu}_{\ell}\right)$, JHEP 11 (2019) 001 [arXiv:1907.09359] [INSPIRE].

[63] S. Catani and M.H. Seymour, A general algorithm for calculating jet cross-sections in NLO QCD, Nucl. Phys. B 485 (1997) 291 [Erratum ibid. B 510 (1998) 503] [hep-ph/9605323] [INSPIRE].

[64] S. Catani, S. Dittmaier, M.H. Seymour and Z. Trócsányi, The dipole formalism for next-to-leading order QCD calculations with massive partons, Nucl. Phys. B 627 (2002) 189 [hep-ph/0201036] [INSPIRE].

[65] J.M. Campbell, R.K. Ellis and F. Tramontano, Single top production and decay at next-to-leading order, Phys. Rev. D 70 (2004) 094012 [hep-ph/0408158] [INSPIRE].

[66] Z. Nagy and Z. Trócsányi, Next-to-leading order calculation of four jet observables in electron positron annihilation, Phys. Rev. D 59 (1999) 014020 [Erratum ibid. D 62 (2000) 099902] [hep-ph/9806317] [INSPIRE].

[67] Z. Nagy, Next-to-leading order calculation of three jet observables in hadron hadron collision, Phys. Rev. D 68 (2003) 094002 [hep-ph/0307268] [INSPIRE].

[68] S. Dulat et al., New parton distribution functions from a global analysis of quantum chromodynamics, Phys. Rev. D 93 (2016) 033006 [arXiv:1506.07443] [INSPIRE].

[69] NNPDF collaboration, Parton distributions for the LHC Run II, JHEP 04 (2015) 040 [arXiv: 1410.8849$]$ [INSPIRE].

[70] L.A. Harland-Lang, A.D. Martin, P. Motylinski and R.S. Thorne, Parton distributions in the LHC era: MMHT 2014 PDFs, Eur. Phys. J. C 75 (2015) 204 [arXiv:1412.3989] [InSPIRE].

[71] M. Cacciari, G.P. Salam and G. Soyez, The anti-k $k_{t}$ jet clustering algorithm, JHEP 04 (2008) 063 [arXiv: 0802.1189] [INSPIRE].

[72] S. Frixione, Isolated photons in perturbative QCD, Phys. Lett. B 429 (1998) 369 [hep-ph/9801442] [INSPIRE].

[73] M. Jezabek and J.H. Kuhn, QCD corrections to semileptonic decays of heavy quarks, Nucl. Phys. B 314 (1989) 1 [INSPIRE]. 
[74] K.G. Chetyrkin, R. Harlander, T. Seidensticker and M. Steinhauser, Second order QCD corrections to $\Gamma(t \rightarrow W b)$, Phys. Rev. D 60 (1999) 114015 [hep-ph/9906273] [INSPIRE].

[75] A. Denner, S. Dittmaier, S. Kallweit and S. Pozzorini, NLO QCD corrections to off-shell top-antitop production with leptonic decays at hadron colliders, JHEP 10 (2012) 110 [arXiv: 1207.5018] [INSPIRE].

[76] J. Butterworth et al., PDF4LHC recommendations for LHC Run II, J. Phys. G 43 (2016) 023001 [arXiv: 1510.03865] [INSPIRE].

[77] G. Heinrich, A. Maier, R. Nisius, J. Schlenk and J. Winter, NLO QCD corrections to $W^{+} W^{-} b \bar{b}$ production with leptonic decays in the light of top quark mass and asymmetry measurements, JHEP 06 (2014) 158 [arXiv: 1312.6659] [INSPIRE].

[78] G. Heinrich et al., NLO and off-shell effects in top quark mass determinations, JHEP 07 (2018) 129 [arXiv:1709.08615] [inSPIRE].

[79] S. Ferrario Ravasio, T. Ježo, P. Nason and C. Oleari, A theoretical study of top-mass measurements at the LHC using $N L O+P S$ generators of increasing accuracy, Eur. Phys. J. C 78 (2018) 458 [arXiv:1801.03944] [INSPIRE].

[80] M. Beneke et al., Top quark physics, in the proceedings of the 1999 CERN Workshop on standard model physics (and more) at the LHC, May 25-26, CERN, Geneva, Switzerland (2000), hep-ph/0003033 [INSPIRE].

[81] S. Liebler, G. Moortgat-Pick and A.S. Papanastasiou, Probing the top-quark width through ratios of resonance contributions of $e^{+} e^{-} \rightarrow W^{+} W^{-} b \bar{b}$, JHEP 03 (2016) 099 [arXiv: 1511.02350] [INSPIRE].

[82] A. Baskakov, E. Boos and L. Dudko, Model independent top quark width measurement using a combination of resonant and nonresonant cross sections, Phys. Rev. D 98 (2018) 116011 [arXiv: 1807.11193] [INSPIRE]. 